\title{
Supernovae in Early\#Type Galaxies: Directly Connecting Age and Metallicity with Type la Luminosity
}

\section{Citation}

Gallagher, Joseph S., Peter M. Garnavich, Nelson Caldwell, Robert P. Kirshner, Saurabh W. Jha, Weidong Li, Mohan Ganeshalingam, and Alexei V. Filippenko. 2008. "Supernovae in Early\#Type Galaxies: Directly Connecting Age and Metallicity with Type la Luminosity." The Astrophysical Journal 685 (2): 752-66. https://doi.org/10.1086/590659.

\section{Permanent link}

http://nrs.harvard.edu/urn-3:HUL.InstRepos:41399732

\section{Terms of Use}

This article was downloaded from Harvard University's DASH repository, and is made available under the terms and conditions applicable to Other Posted Material, as set forth at http:// nrs.harvard.edu/urn-3:HUL.InstRepos:dash.current.terms-of-use\#LAA

\section{Share Your Story}

The Harvard community has made this article openly available.

Please share how this access benefits you. Submit a story.

\section{Accessibility}


The Astrophysical Journal, 685:752-766, 2008 October 1

(C) 2008. The American Astronomical Society. All rights reserved. Printed in U.S.A.

\title{
SUPERNOVAE IN EARLY-TYPE GALAXIES: DIRECTLY CONNECTING AGE AND METALLICITY WITH TYPE Ia LUMINOSITY
}

\author{
Joseph S. Gallagher and Peter M. Garnavich \\ Department of Physics, University of Notre Dame, 225 Nieuwland Science Hall, Notre Dame, IN 46556-5670; jgallag3@nd.edu \\ Nelson Caldwell and Robert P. Kirshner \\ Harvard-Smithsonian Center for Astrophysics, 60 Garden Street, Cambridge, MA 02138 \\ SAURABH W. JHA \\ Department of Physics and Astronomy, Rutgers, The State University of New Jersey, 136 Frelinghuysen Road, Piscataway, NJ 08854
}

AND

Weidong Li, Mohan Ganeshalingam, and Alexei V. Filippenko

Department of Astronomy, University of California, Berkeley, CA 94720-3411

Received 2008 April 28; accepted 2008 May 30

\begin{abstract}
We have obtained optical spectra of 29 early-type (E/S0) galaxies that hosted Type Ia supernovae (SNe Ia). We have measured absorption-line strengths and compared them to a grid of models to extract the relations between the supernova properties and the luminosity-weighted age/composition of the host galaxies. Such a direct measurement is a marked improvement over existing analyses that tend to rely on general correlations between the properties of stellar populations and morphology. We find a strong correlation suggesting that $\mathrm{SNe}$ Ia in galaxies whose populations have a characteristic age greater than $5 \mathrm{Gyr}$ are $\sim 1 \mathrm{mag}$ fainter at $V_{\max }$ than those found in galaxies with younger populations. We find that SN Ia distance residuals in the Hubble diagram are correlated with host-galaxy metal abundance with higher iron abundance galaxies hosting less-luminous supernovae. We thus conclude that the time since progenitor formation primarily determines the radioactive Ni production while progenitor metal abundance has a weaker influence on peak luminosity, but one not fully corrected by light-curve shape and color fitters. This result, particularly the secondary dependence on metallicity, has significant implications for the determination of the equationof-state parameter, $w=P /\left(\rho c^{2}\right)$, and could impact planning for future dark-energy missions such as JDEM. Assuming no selection effects in discovering SNe Ia in local early-type galaxies, we find a higher specific SN Ia rate in E/S0 galaxies with ages below $3 \mathrm{Gyr}$ than in older hosts. The higher rate and brighter luminosities seen in the youngest E/S0 hosts may be a result of recent star formation and represents a tail of the "prompt" SN Ia progenitors.
\end{abstract}

Subject headings: cosmology: observations — galaxies: elliptical and lenticular, cD galaxies: fundamental parameters - supernovae: general

\section{INTRODUCTION}

Type Ia supernovae (SNe Ia) have proven to be effective standardizable candles, and their high peak luminosity has made them excellent cosmological probes. An empirically determined relation between light-curve properties and peak absolute magnitude (Phillips 1993; Hamuy et al. 1996; Riess et al. 1996; Goldhaber et al. 2001; Guy et al. 2007; Jha et al. 2007; Conley et al. 2008) has yielded improved estimates of the global Hubble constant (Jha et al. 1999; Freedman et al. 2001; Riess et al. 2005; Sandage et al. 2006). Although the earliest attempts to measure the matter density of the universe preceded these light-curve shape methods (Norgaard-Neilsen et al. 1989), and the initial efforts by the Supernova Cosmology Project (SCP) led to incorrect conclusions about the cosmic matter density (Perlmutter et al. 1997), early work by both the SCP and the High-Z Supernova Search Team (Schmidt et al. 1998) found evidence for low matter density (Perlmutter et al. 1998; Garnavich et al. 1998a).

Subsequent publications by Riess et al. (1998) and Perlmutter et al. (1999) came to the surprising conclusion that the universe is expanding at an accelerating rate, driven by a mysterious dark energy; see Kirshner (2002), Filippenko (2005b), or Frieman et al. (2008) for reviews. This surprising result has been confirmed by more recent supernova observations (Knop et al. 2003; Tonry et al. 2003; Barris et al. 2004; Riess et al. 2004) and by comple- mentary measurements of the cosmic microwave background (CMB) anisotropies (e.g., Bennett et al. 2003) and large-scale structure (e.g., Peacock et al. 2001). Focus has now shifted from demonstrating the existence of dark energy to constraining its properties (Garnavich et al. 1998b; Astier et al. 2006; Riess et al. 2007; Wood-Vasey et al. 2007; Miknaitis et al. 2007; Eisenstein et al. 2007; Frieman et al. 2007) and sharpening the tools available for its study (e.g., Krisciunas et al. 2007; Wood-Vasey et al. 2008).

Analyses of their spectra and light curves have led to the consensus that $\mathrm{SNe}$ Ia are well characterized by a thermonuclear disruption of a carbon-oxygen white dwarf (WD). The transition from a dynamically stable WD to an explosion capable of outshining an entire galaxy is precipitated by the WD mass approaching the Chandrasekhar mass limit through matter accretion from a close binary companion. The nature of this accretion process is still uncertain, although two scenarios have become the focus of $\mathrm{SN}$ Ia progenitor modeling. The first is the single-degenerate (SD) model in which a WD accretes matter from a nondegenerate companion (Whelan \& Iben 1973; Nomoto 1982), while the second is the double-degenerate (DD) model involving the merger to two WDs (Iben \& Tutukov 1984; Webbink 1984). Although promising, our understanding of these models remains incomplete, with questions persisting about the nature of the binary companion (main sequence, asymptotic giant branch, etc.) and accretion 
flow in the SD scenario, the apparent likelihood that a WD merger will result in an accretion-induced collapse rather than an SN Ia, and the potential for multiple stellar population-dependent channels leading to SN Ia explosions.

Differentiating among dark-energy models requires mapping the expansion history of the universe with high precision and a small systematic error. Applying SNe Ia to this problem is complicated by the uncertain nature of the explosion physics and progenitor stars (e.g., Howell et al. 2006; Hicken et al. 2007). Even with the most basic assumption, that an SN Ia results from the thermonuclear disruption of a near-Chandrasekhar mass carbonoxygen WD, there remain potential dangers in blindly applying SNe Ia to the dark-energy problem. Theoretical models (Höflich et al. 1998; Umeda et al. 1999; Timmes et al. 2003) suggest that population age and metallicity may have an effect on the WD composition and influence the SN Ia peak luminosity. Since the average age and metal content of stars have evolved over cosmic time, the ensemble character of SN Ia explosions may be a function of look-back time. But the local universe contains a range of stellar ages and metallicities, so an empirical calibration of these effects may be possible.

Hamuy et al. (1996) were the first to note a correlation between SN Ia decline rate and host-galaxy morphology. The results showed that intrinsically faint events occur in early-type (E/S0) galaxies, while luminous events are often hosted by late-type galaxies. This correlation with morphology has since been confirmed in larger SN Ia samples (e.g., Gallagher et al. 2005a), but the cause of the trend remains unknown. The existence of a correlation is important because it demonstrates that the SN Ia environment imprints itself on some aspect of the progenitor. On average, spiral and elliptical galaxies differ in population age, star formation rate (SFR), and metal content, with significant overlap in these properties across the morphological types. Hamuy et al. (2000) measured the metallicity of a small sample of star-forming hosts to test if age or metal content was the factor influencing SN Ia luminosity, but the results were ambiguous.

Gallagher et al. (2005a) expanded the number of observed hosts to test the impact of stellar environment on the photometric properties of nearby SNe Ia. Potential dependencies of SN decline rate on host-galaxy absolute magnitude and star formation history were investigated in a sample of galaxies with a broad range of properties and across the full Hubble sequence. Metallicity was measured from emission lines, limiting this aspect of the study to star-forming hosts. No significant correlation between metallicity and decline rate was detected, although a tenuous correlation was found between the metal content and Hubble residuals. Furthermore, only galaxies without significant star formation were found to host faint, fast-declining SNe Ia. The division by star formation history was more stark than by morphology alone, suggesting that the observed luminosity spread in SNe Ia results from the influence of population age on the WD progenitors. The luminosity/star formation relation has also been seen in a highredshift sample of SNe Ia studied by the Supernova Legacy Survey (SNLS; Sullivan et al. 2006).

The specific SN Ia rate is strongly dependent on the amount of current star formation (Mannucci et al. 2005; Sullivan et al. 2006). Actively star-forming galaxies host approximately 10 times as many SNe Ia per unit mass than do their low star-forming counterparts. Scannapieco \& Bildsten (2005) find that the SN Ia rate is well described by a combination of two distinct populations of SNe Ia: a "prompt" (shorter delay time) SN Ia component dependent on the recent star formation in the galaxy and a "delayed" or "tardy" (longer delay time) component dependent on the number of low-mass stars. Assuming the light-curve shape correction methods are perfect, then this will not have an effect on the recent cosmological results. However, if the corrected photometric properties of these two SN populations differ (e.g., a variation due to age or metallicity), then it could have implications for derived cosmological parameters because the short delay time SNe are expected to dominate at high redshift whereas the long delay time events are expected to dominate at low redshift.

Here we present our study on the luminosity-weighted ages and metal abundances of early-type host galaxies. Early-type galaxies, unlike star-forming spirals, have been shown to host both faint and bright $\mathrm{SNe}$ Ia, thereby allowing us to sample the full diversity of SNe Ia. Consequently, we focus our study on absorption-line spectra of host galaxies and utilize single age, single metallicity stellar population models to characterize the stellar populations (Vazdekis et al. 1996, 2003, 2008, in preparation; Vazdekis 1999; Blakeslee et al. 2001). The impact of both age and metallicity on SN peak magnitude is studied, and the sample age and metallicity distributions will be compared to absorption-line spectra of field galaxies obtained from the Sloan Digital Sky Survey (SDSS). Furthermore, we will infer from our data a measurement of the relative SN Ia rate as a function of galaxy age. Finally, we will search for second-order dependencies at the level of the SN Ia intrinsic scatter by comparing host-galaxy ages and metallicities to the SN residuals from the Hubble diagram.

We detail our observational techniques and data-reduction pipeline in $\S 2$ of this paper. In $\S 3$ we report the methods for performing emission-line corrections, and for obtaining age and metallicity estimates of our host sample. Our results are presented in $\S 4$, and the summary and conclusions are in $\S 5$.

\section{OBSERVATIONS AND DATA REDUCTION}

\subsection{Observations}

The objective of this study was to obtain absorption-line spectra of SN Ia host galaxies with the intent of estimating their ages and metallicities through a comparison with the simple stellar population (SSP) models of Alexandre Vazdekis (A. Vazdekis et al. 2008, in preparation; Sanchez-Blazquez et al. 2006). To this end, we obtained spectra of a sample of SN Ia host galaxies with the $1.5 \mathrm{~m}$ Tillinghast telescope located at the F. L. Whipple Observatory. We employed the FAST spectrograph (Fabricant et al. 1998) fitted with the 300 line $\mathrm{mm}^{-1}$ reflection grating and a $3^{\prime \prime} \times 3^{\prime}$ slit. The setup gave a resolution of $\sim 7 \AA$ full width at halfmaximum (FHWM) intensity.

The spectra were obtained over the course of six nights during 2005 April and July. Our set was compiled from the host galaxies of SNe Ia from the samples of Phillips et al. (1999), Jha (2002), and M. Ganeshalingam et al. (2008, in preparation). Suitable targets were those classified as having early-type ( $\mathrm{S} 0-\mathrm{E})$, as well as spiral galaxies containing strong absorption lines in their spectra (and minimal emission lines) as determined by Gallagher et al. (2005a). In addition to the host galaxies, we also obtained spectra for a set of comparison elliptical galaxies having age and metallicity estimates determined by Trager et al. (2000).

The slit was aligned along the galaxy's major axis with position angles (P.A.s) determined via the Digital Sky Survey (DSS) plates. In a few cases the P.A. was altered to prevent a nearby star from falling on the slit. Exposure times were varied depending on the galaxy brightness, with a target signal-to-noise ratio $(\mathrm{S} / \mathrm{N})$ of 10-20. For each target we obtained three spectra that we subsequently combined to improve the $\mathrm{S} / \mathrm{N}$ and remove cosmic rays. Bias, dark, and dome-flat exposures were taken at the beginning and the end of each night, and a comparison spectrum of a He-Ne-Ar lamp was acquired before each target for wavelength calibration. 
TABLE 1

Absorption-Line Galaxy Sample

\begin{tabular}{|c|c|c|c|c|c|c|}
\hline $\begin{array}{c}\text { Galaxy } \\
\text { (1) }\end{array}$ & $\begin{array}{l}\mathrm{SN} \\
(2)\end{array}$ & $\begin{array}{r}M_{V} \\
(3)\end{array}$ & $\begin{array}{c}\text { Morphology } \\
\text { (4) }\end{array}$ & $\begin{array}{l}\text { P.A. } \\
\text { (deg) } \\
(5)\end{array}$ & $\begin{array}{l}\text { Approx Width } \\
\text { (arcsec) } \\
(6)\end{array}$ & $\begin{array}{c}\text { L.C. Ref. } \\
\text { (7) }\end{array}$ \\
\hline \multicolumn{7}{|c|}{ SN Ia Host-Galaxy Sample } \\
\hline 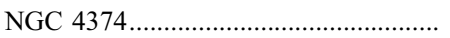 & $1991 \mathrm{bg}$ & -17.88 & E1 & 90 & 9.63 & 1 \\
\hline NGC $4526 \ldots \ldots$. & 1994D & -18.98 & So & 110 & 10.78 & 1 \\
\hline …....................... & 1994M & -19.09 & $\mathrm{E}$ & 40 & 4.03 & 1 \\
\hline 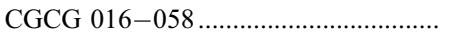 & $1994 \mathrm{~T}$ & -18.67 & $\mathrm{Sa}$ & -30 & 3.01 & 1 \\
\hline$\ldots \ldots \ldots \ldots \ldots \ldots \ldots \ldots \ldots \ldots$ & 1995D & -19.42 & $\mathrm{~S} 0+$ & 0 & 6.90 & 1 \\
\hline 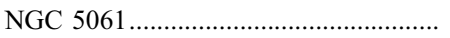 & 1996X & -19.21 & $\mathrm{Sa}$ & 90 & 4.17 & 1 \\
\hline 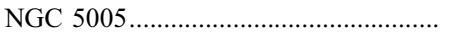 & 1996ai & -19.58 & Sbc & -30 & 12.66 & 1 \\
\hline 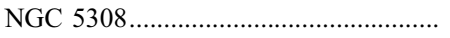 & 1996bk & -18.33 & $\mathrm{~S} 0-$ & 60 & 10.78 & 1 \\
\hline 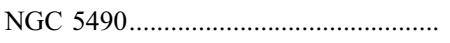 & $1997 \mathrm{cn}$ & -17.87 & $\mathrm{E}$ & 0 & 5.17 & 1 \\
\hline 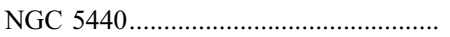 & 1998D & $\ldots$ & $\mathrm{Sa}$ & 40 & 6.19 & $\ldots$ \\
\hline UGC $11149 \ldots$. & $1998 d x$ & -19.14 & $\mathrm{Sab}$ & -40 & 6.75 & 1 \\
\hline 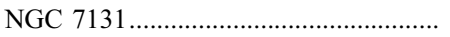 & $1998 \mathrm{co}$ & $\ldots$ & S0 & -35 & 4.45 & 1 \\
\hline 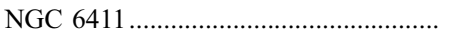 & 1999da & -17.81 & $\mathrm{E}$ & 70 & 8.19 & 1 \\
\hline 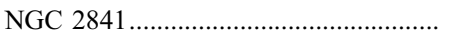 & 1999by & -17.95 & $\mathrm{Sab}$ & 50 & 5.88 & 1 \\
\hline 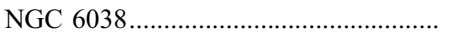 & $1999 \mathrm{cc}$ & -19.00 & $\mathrm{Sc}$ & 105 & 8.19 & 1 \\
\hline 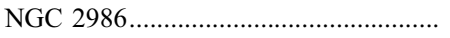 & $1999 \mathrm{gh}$ & -18.43 & E2; HII & 10 & 6.90 & 1 \\
\hline 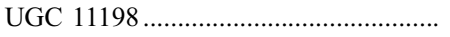 & $2000 \mathrm{dm}$ & -18.94 & $\mathrm{Sab}$ & 45 & 7.06 & 2 \\
\hline 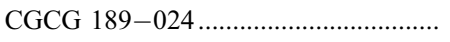 & $2002 \mathrm{G}$ & -19.347 & $\mathrm{E}$ & 10 & 4.16 & 2 \\
\hline 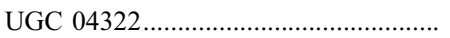 & 2002he & -19.01 & $\mathrm{E}$ & 40 & 5.32 & 2 \\
\hline 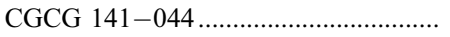 & $2001 b f$ & -19.514 & $\ldots$ & 100 & 5.04 & 2 \\
\hline Anon & 2002aw & -19.35 & $\mathrm{Sb}(\mathrm{f})$ & 80 & 13.94 & 2 \\
\hline 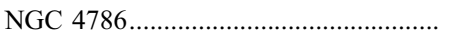 & $2002 \mathrm{cf}$ & -17.82 & $\mathrm{E}+$ pec & -20 & 6.47 & 2 \\
\hline 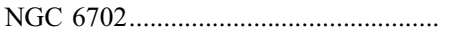 & $2002 \mathrm{cs}$ & $\ldots$ & $\mathrm{E}$ & 55 & 6.31 & $\ldots$ \\
\hline 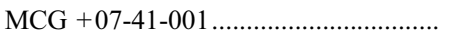 & 2002 do & -18.48 & E1 & 0 & 20.85 & 2 \\
\hline 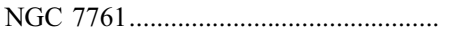 & 2002ef & -19.30 & So & 90 & 4.61 & 2 \\
\hline 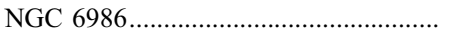 & 2002el & -19.11 & SB0- & 5 & 5.89 & 2 \\
\hline 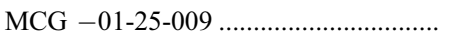 & 2003D & -18.31 & E1 & 10 & 5.18 & 2 \\
\hline IC 0522 & $2003 Y$ & $\ldots$ & So & -20 & 7.34 & $\ldots$ \\
\hline 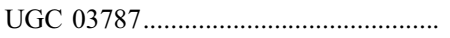 & $2003 \mathrm{ch}$ & $\ldots$ & E-S0 & 0 & 4.47 & $\ldots$ \\
\hline 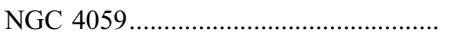 & $2005 \mathrm{bl}$ & -17.62 & $\mathrm{E}$ & 40 & 4.61 & 3 \\
\hline
\end{tabular}

Trager et al. (2000) Comparison Sample

\begin{tabular}{|c|c|c|c|c|c|c|}
\hline 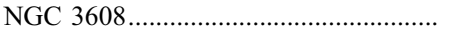 & $\ldots$ & $\ldots$ & E2 & 80 & 6.19 & $\ldots$ \\
\hline NGC $4472 \ldots \ldots \ldots . .$. & $\ldots$ & $\ldots$ & $\mathrm{E} 2 / \mathrm{S} 0$ & -20 & 12.80 & $\ldots$ \\
\hline 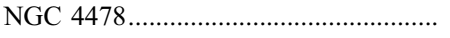 & $\ldots$ & $\ldots$ & E2 & 40 & 6.17 & $\ldots$ \\
\hline NGC $4489 . . . \ldots \ldots . . . .$. & $\ldots$ & $\ldots$ & $\mathrm{E}$ & 110 & 4.61 & $\ldots$ \\
\hline 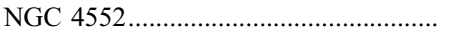 & $\ldots$ & $\ldots$ & E; HII & -30 & 6.31 & $\ldots$ \\
\hline 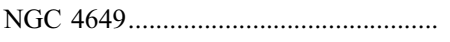 & $\ldots$ & $\ldots$ & E2 & 90 & 13.09 & $\ldots$ \\
\hline 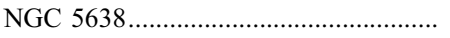 & $\ldots$ & $\ldots$ & E1 & -20 & 8.04 & $\ldots$ \\
\hline 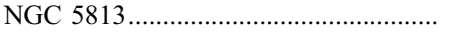 & $\cdots$ & $\cdots$ & $\mathrm{E} 1-2$ & -20 & 6.17 & $\ldots$ \\
\hline 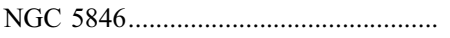 & $\ldots$ & $\ldots$ & $\mathrm{E} 0-1$ & 50 & 11.35 & $\ldots$ \\
\hline 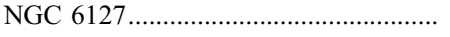 & $\cdots$ & $\cdots$ & $\mathrm{E}$ & 90 & 5.32 & $\ldots$ \\
\hline 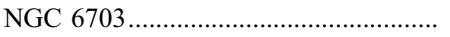 & $\ldots$ & $\ldots$ & $\mathrm{SA} 0-$ & 90 & 5.17 & $\ldots$ \\
\hline
\end{tabular}

(1) Jha et al. 2007; (2) Ganeshalingam et al. 2008; Filippenko et al. 2001; Filippenko 2005a; (3) Taubenberger et al. 2008; Gallagher et al. $2005 \mathrm{~b}$.

Finally, flux standard star spectra were taken each night with the slit oriented along the parallactic angle (Filippenko 1982).

Our full sample of galaxies can be seen in Table 1. Columns (1) and (2) give the host-galaxy name and hosted SN, respectively. Column (3) provides the peak magnitudes of the hosted SN Ia, determined from the SN light-curve data and employing MLCS2k2 (Jha et al. 2007). Column (4) gives the morphological classification of each galaxy, while column (5) reports the P.A. for each observation. Column (6) shows the width of the extracted aperture encompassing all the light out to approximately the effective radius, $R_{e}{ }^{1}$ Finally, column (7) gives the source of the SN light curve: (1) the CfA sample (Jha et al. 2007), (2) the Katzman Automatic

\footnotetext{
${ }^{1}$ The radius within which half of the total galactic light is contained.
}

Imaging Telescope (KAIT; Ganeshalingam et al. 2008; Filippenko et al. 2001; Filippenko 2005a), and (3) SN 2005bl ( Taubenberger et al. 2008; Gallagher et al. 2005b).

\subsection{Data Reduction}

The majority of data reduction for this study was performed using standard techniques within IRAF. ${ }^{2}$ The data were bias- and

\footnotetext{
${ }^{2}$ IRAF is the Image Reduction and Analysis Facility, a general purpose software system for the reduction and analysis of astronomical data. IRAF is written and supported by the IRAF programming group at the National Optical Astronomy Observatories (NOAO) in Tucson, AZ. NOAO is operated by the Association of Universities for Research in Astronomy (AURA), Inc., under cooperative agreement with the National Science Foundation (NSF).
} 
dark-subtracted, and flat-fielded to remove the pixel-to-pixel variations in the CCD; also, the bad rows and columns of the CCD were interpolated using the routine FIXPIX. Following preprocessing, the task was to combine the set of three spectral images that we had for each target. The first step was the removal of cosmic rays. In the interest of preserving every image in a set, thereby increasing our statistics, we removed each cosmic ray individually using the IMEDIT routine in IRAF. Cosmic rays that fell on the galaxy nucleus itself were left alone until after the aperture extraction.

One-dimensional aperture extraction was performed using the APALL routine with extraction radii equal to the effective radius $\left(R_{e}\right)$ of each galaxy. The effective radii were determined through an IDL code written to integrate over the average profile of 400 centrally located columns along the spatial axis of each image. Each spectrum was then individually checked for cosmic rays landing on one of our relevant absorption-line indices or the surrounding continua. In the event that an interfering cosmic ray was found, steps were taken to either remove it interactively within SPLOT, or to remove the image altogether if the cosmic ray was too disruptive. The average of each set of images was generated using IM-COMBINE, and the resultant spectra were wavelength- and flux-calibrated. Finally, the spectra were corrected for Galactic extinction using the IRAF routine DEREDDEN (Cardelli et al. 1989).

\section{DISENTANGLING AGE AND METALLICITY}

\subsection{Background}

A well-known and much reviled puzzle in the study of stellar populations is the observed similarity between the effects of age and metallicity on the integrated light of stellar populations (e.g., Worthey 1994). Astronomers attempt to unravel this age/ metallicity degeneracy through the development of stellar population models. The simplified goal of stellar population synthesis modeling is to find a combination of stars for which the integrated spectrum of the stars matches the observed spectrum of the population under study.

Early empirical techniques such as quadratic programming devised by Faber (1972) have given way to evolutionary population synthesis models that have been improving since the 1970 s (Tinsley \& Gunn 1976; Tinsley 1980; Gunn et al. 1981; Worthey 1994; Buzzoni 1995; Bressan et al. 1996; Maraston 1998; Bruzual \& Charlot 2003; Fioc \& Rocca-Volmerange 1997; Vázquez \& Leitherer 2005; Vazdekis et al. 2003; González Delgado et al. 2005). Whereas empirical models are constructed from a combination of stellar spectra or the spectra of stellar clusters, evolutionary synthesis models utilize theoretical stellar evolutionary isochrones as the primary constituent. A single isochrone on the Hertzsprung-Russell (HR) diagram gives the locus of luminosities and temperatures at a single moment in time for stars of all masses. Together with an assumed initial mass function (IMF), an isochrone can be built to accurately model a single-age stellar population. The final model is obtained by converting isochrone parameters to observed spectra and finally integrating along the isochrone.

The populations that are modeled using the above techniques are known as simple stellar populations, or SSPs. SSPs are, by definition, a population of stars created during a single burst event (i.e., all the stars have the same age) and possessing a single global metallicity. However, early-type galaxies are often composed of multiple-component stellar populations. Galaxy ages derived through a comparison with these single-component SSPs are luminosity-weighted and are typically sensitive to the youngest component in a real early-type galaxy. Consequently, it is ap- propriate to view the ages derived in studies such as ours as lower limit estimates (Renzini 2006).

The determination of age and metallicity via galactic spectra is fairly simple, in principle. We can exploit predictions made by population synthesis models to relate physical properties, such as age and metallicity, to observables, such as the absorption-line strengths for the strongest atomic and molecular absorption features in the optical range. Measuring the relative strengths of these absorption features, we can estimate the age and metallicity of the stellar population that created the spectrum.

\subsection{Model Broadening}

For the age and metallicity estimates of our galaxies, we employ the single age, single metallicity stellar population models described by Vazdekis et al. (1996, 2003; A. Vazdekis et al. 2008, in preparation), Vazdekis (1999), and Blakeslee et al. (2001). The models make use of the empirical stellar spectral library MILES (Sanchez-Blazquez et al. 2006; Cenarro et al. 2007). MILES is a marked improvement over previous libraries; it has 985 stars, a spectral resolution of $2.3 \AA$ (FWHM), and a wavelength range of 3525-7500 $\AA$.

One advantage of empirical libraries over theoretical stellar libraries is that they are based on actual stellar spectra and are not dependent on the potential inaccuracies and underlying assumptions inherent in any theoretical model. However, in order for an empirical stellar library to sufficiently cover the parameter space in temperature, gravity, abundance, and $[\alpha / \mathrm{Fe}]$, the stellar observations must be of the highest quality. Consequently, empirical stellar libraries have traditionally been restricted to the nearest stars, thereby introducing a bias of atmospheric parameters toward those that are seen in the solar neighborhood. The MILES library acquired atmospheric parameters from the literature and calibrated the set through a subset of reference field stars from Soubiran et al. (1998); for full details see Cenarro et al. (2007). In this way, MILES has optimized its stellar atmosphere coverage and improved on previous libraries. Nevertheless, because earlytype galaxies are generally $\alpha$-enhanced and metal-rich, the potential bias toward solar abundance ratios should be kept in mind.

The stellar population synthesis models derived from MILES consist of single age, single metallicity spectral energy distributions (SEDs) that adopt a standard Salpeter IMF (Salpeter 1955). The SEDs possess a FWHM resolution of $2.3 \AA$ and a spectral range similar to our data at $3540-7410 \AA$. There are 276 models with ages ranging from 0.10 to $17.78 \mathrm{Gyr}$ and metallicities ranging from $[\mathrm{M} / \mathrm{H}]=-1.68$ to 0.20 .

In the past, the most widely used method of estimating stellar population age and metallicity has been through a comparison of absorption-line indices with those of the Lick/IDS System (Worthey 1994). Since the stars of the Lick/IDS stellar library are not flux-calibrated, data must be converted to the instrumental response curve of the original data set (Worthey \& Ottaviani 1997). With the advent of improved, flux-calibrated stellar libraries, it became possible to generate complete SEDs of a single stellar population (Jones 1997; Sanchez-Blazquez et al. 2006; Vazdekis \& Arimoto 1999), thereby eliminating the need for such a correction. Furthermore, in order to use the Lick population models, it is necessary to transform the observational data to match the resolution of the Lick spectrograph $(\sim 8-10 \AA$ FWHM). For our analysis, the model SEDs are at a higher resolution than the data; consequently, the models were required to be broadened to the resolution of our data rather than the other way around.

Thus, the first step in the analysis was to characterize the wavelength-dependent resolution of our spectra. This was accomplished by measuring the line widths of emission lines in the 
TABLE 2

Cross-Correlation Results

\begin{tabular}{|c|c|c|c|}
\hline $\begin{array}{l}\text { Galaxy } \\
\text { (1) }\end{array}$ & $\begin{array}{c}v_{r} \\
\left(\mathrm{~km} \mathrm{~s}^{-1}\right) \\
(2)\end{array}$ & $\begin{array}{c}\delta v_{r} \\
\left(\mathrm{~km} \mathrm{~s}^{-1}\right) \\
(3)\end{array}$ & $\begin{array}{c}\sigma \\
\left(\mathrm{km} \mathrm{s}^{-1}\right) \\
(4)\end{array}$ \\
\hline Anon.... & 7932.43 & 19.66 & 136.91 \\
\hline CGCG $016-058 \ldots \ldots$ & 10411.31 & 17.79 & 147.99 \\
\hline CGCG $141-044 \ldots \ldots \ldots \ldots \ldots . . .$. & 4665.13 & 23.01 & 109.73 \\
\hline CGCG 189-024 ....................... & 10113.14 & 21.01 & 188.70 \\
\hline IC 0522 & 5092.26 & 19.05 & 170.76 \\
\hline MCG + 07-41-001 …................. & 4584.86 & 68.68 & 344.72 \\
\hline MCG -01-25-009. & 6623.73 & 21.58 & 251.97 \\
\hline 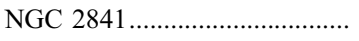 & 633.49 & 25.43 & 244.72 \\
\hline 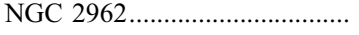 & 1967.99 & 19.01 & 186.61 \\
\hline 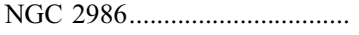 & 2310.81 & 33.56 & 283.67 \\
\hline NGC $3608 \ldots \ldots \ldots \ldots \ldots \ldots \ldots$ & 1237.80 & 21.24 & 209.54 \\
\hline 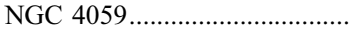 & 7195.99 & 33.21 & 210.88 \\
\hline NGC $4374 \ldots \ldots$. & 1020.48 & 30.57 & 316.56 \\
\hline NGC $4472 \ldots$ & 957.20 & 30.95 & 309.86 \\
\hline NGC 4478 & 1352.18 & 17.67 & 183.09 \\
\hline NGC $4489 \ldots$. & 948.10 & 11.75 & 106.89 \\
\hline NGC $4493 \ldots . .$. & 6957.26 & 20.98 & 214.39 \\
\hline NGC $4526 \ldots \ldots$. & 604.65 & 24.90 & 235.75 \\
\hline NGC $4552 \ldots$ & 316.64 & 27.18 & 282.82 \\
\hline NGC 4649 & 1087.03 & 37.32 & 344.45 \\
\hline 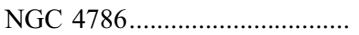 & 4611.97 & 33.49 & 288.86 \\
\hline NGC $5005 \ldots \ldots \ldots$ & 932.64 & 23.29 & 207.73 \\
\hline 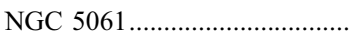 & 2059.50 & 22.15 & 218.96 \\
\hline 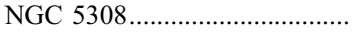 & 2014.72 & 22.08 & 241.36 \\
\hline 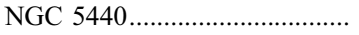 & 3697.03 & 27.35 & 238.53 \\
\hline 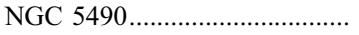 & 4965.75 & 37.73 & 338.21 \\
\hline NGC 5638 & 1640.05 & 20.15 & 172.96 \\
\hline 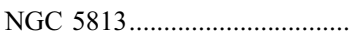 & 1948.27 & 28.57 & 248.58 \\
\hline 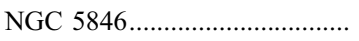 & 1692.00 & 27.71 & 238.42 \\
\hline 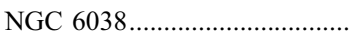 & 9399.72 & 32.83 & 183.91 \\
\hline NGC $6127 \ldots \ldots \ldots$ & 4715.52 & 29.99 & 264.50 \\
\hline 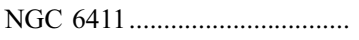 & 3726.29 & 23.52 & 186.15 \\
\hline NGC $6702 \ldots \ldots$ & 4721.40 & 32.71 & 209.44 \\
\hline 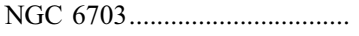 & 2384.69 & 20.62 & 199.69 \\
\hline 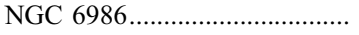 & 8534.95 & 25.52 & 267.16 \\
\hline 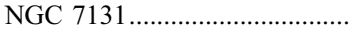 & 5409.40 & 36.77 & 185.25 \\
\hline 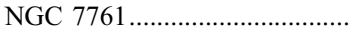 & 7192.42 & 19.90 & 211.39 \\
\hline UGC 11149 & 16137.64 & 92.35 & 146.10 \\
\hline UGC 11198 & 4518.02 & 19.91 & 145.28 \\
\hline UGC $03787 \ldots \ldots \ldots$ & 8612.81 & 57.54 & 140.66 \\
\hline UGC $04322 \ldots \ldots \ldots \ldots$ & 7376.56 & 23.98 & 248.08 \\
\hline
\end{tabular}

He-Ne-Ar lamp across our full spectral range. A polynomial fit to the resulting scatter plot provided a wavelength-dependent resolution function for the FAST spectrograph. We derived a wavelength-dependent broadening term with a square equal to the difference between the square of the host-galaxy resolution and that of the resolution of the Vazdekis models, given as $2.3 \AA$.

The next broadening term we considered was from the velocity dispersion of the host galaxies. To determine the velocity dis- persion for each galaxy, we obtained spectra of velocity standard stars HD 12623 and HD 52071; these were taken within a few months of our observing runs on the Tillinghast telescope using the FAST spectrograph. In total, we obtained six spectra of HD 12623 and 14 spectra of HD 52071. Cross-correlation analysis was then performed between these templates and the host-galaxy spectra using the IRAF routine FXCOR. A velocity dispersion and redshift estimate were obtained using each template-galaxy combination, with the final result for a given host being the average of the measurements made with each of the 20 templates.

Our two broadening terms were summed in quadrature, yielding the final width of our Gaussian kernel used in the convolution with the host galaxy. Each host galaxy had a unique final broadening term, and the Vazdekis model SEDs were broadened independently according to this term. In this way, we created separate age/metallicity grids for each host galaxy. The velocity dispersions and heliocentric velocities resulting from this analysis are shown in Table 2. These heliocentric velocities were converted to CMB rest frame for the subsequent analysis using the NED velocity calculator.

The age-dependent absorption index chosen for this study was $\mathrm{H} \beta . \mathrm{H} \beta$ is known to be metallicity-insensitive, and older stellar populations are known to have relatively strong $\mathrm{H} \beta$ absorption, eliminating the need for high S/N (Cardiel 2003; Caldwell et al. 2003). Although our sample was composed mostly of older stellar populations, there was a chance for $\mathrm{H} \beta$ emission contamination. Consequently, our data were emission-line corrected with the procedure discussed in the next section. We chose two ageinsensitive $\mathrm{Fe}$ absorption lines for our abundance analysis, $\mathrm{Fe} \lambda 5270$ and $\mathrm{Fe} \lambda 4383$. Plotting both of these iron lines against $\mathrm{H} \beta$ produces a strongly orthogonal grid well suited to untangling the age-metallicity degeneracy. Our chosen Lick/IDS indices are summarized in Table 3.

\subsection{Emission-Line Correction}

The presence of $\mathrm{H} \beta$ emission superposed on $\mathrm{H} \beta$ absorption poses a significant problem for our index measurements. The contamination of even weak $\mathrm{H} \beta$ emission lines in our galaxy spectra could significantly decrease the strengths of our measured $\mathrm{H} \beta$ absorption indices, thus systematically biasing our age distribution toward greater ages. Consequently, an emission correction was performed on our host-galaxy spectra. The basic procedure is outlined by Caldwell et al. (2003). The method is based on two main assertions. First, although the underlying absorption-line spectrum at $\mathrm{H} \beta$ is highly sensitive to age, the sensitivity to both age and metallicity is negligible at $\mathrm{H} \alpha$. Second, the observed $\mathrm{Hn} / \mathrm{Fe}$ index ${ }^{3}$ represents an effective indicator of the true underlying absorptionline spectrum, implying that two galaxies with similar $\mathrm{Hn} / \mathrm{Fe}$ indices will likewise possess similar $\mathrm{H} \alpha$ absorption strengths.

Conservatively, we assumed that each galaxy in our sample was contaminated with Balmer emission from $\mathrm{H}$ II regions not

${ }^{3} \mathrm{Hn} / \mathrm{Fe} \equiv\langle\mathrm{H} \delta / \mathrm{Fe} \lambda 4045+\mathrm{H} \gamma / \mathrm{Fe} \lambda 4325+\mathrm{H} 8 / \mathrm{Fe} \lambda 3859\rangle$.

TABLE 3

LICK/IDS INDICES FOR THE STUDY

\begin{tabular}{|c|c|c|c|}
\hline Name & $\begin{array}{c}\text { Index Bandpass } \\
(\AA)\end{array}$ & $\begin{array}{l}\text { Pseudocontinua } \\
\text { (Å) }\end{array}$ & Species Measured \\
\hline $\mathrm{H} \beta$ & $4847.875-4876.625$ & $4827.875-4847.875,4876.625-4891.625$ & $\mathrm{H},(\mathrm{Mg}),(\mathrm{Cr}), \mathrm{C}$ \\
\hline 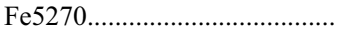 & $5245.650-5285.650$ & $5233.150-5248.150,5285.650-5318.150$ & $\mathrm{Fe}, \mathrm{C},(\mathrm{Mg}), \mathrm{Ca}$ \\
\hline 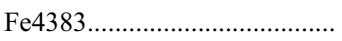 & $4369.125-4420.375$ & $4359.125-4370.375,4442.875-4455.375$ & $\mathrm{Fe}, \mathrm{C},(\mathrm{Si})$ \\
\hline
\end{tabular}




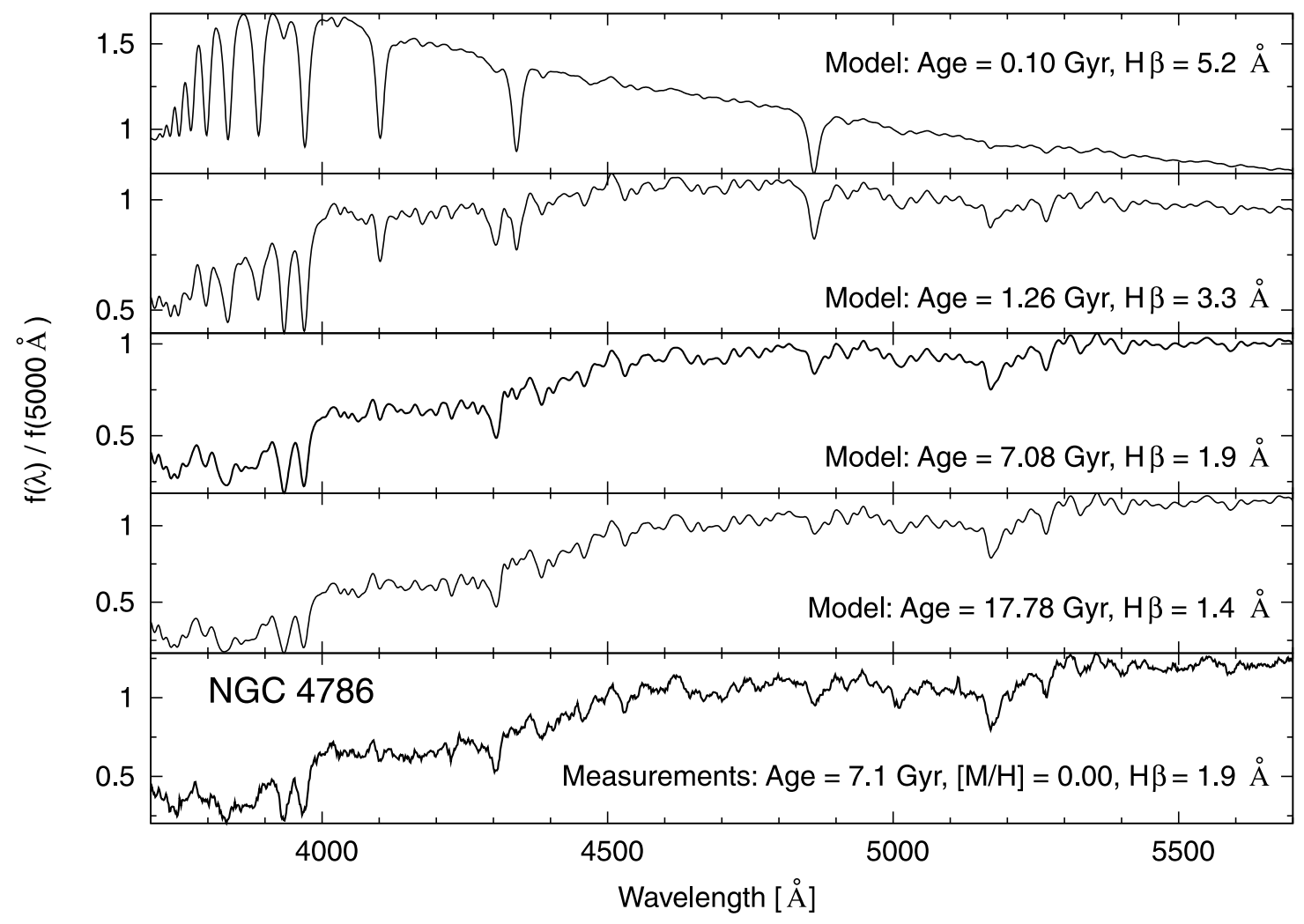

FIG. 1.-Comparison between host-galaxy NGC 4786 with an estimated metallicity around solar and several Vazdekis model SEDs of varying ages. Each model has

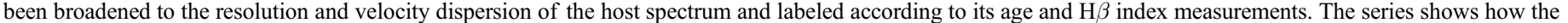

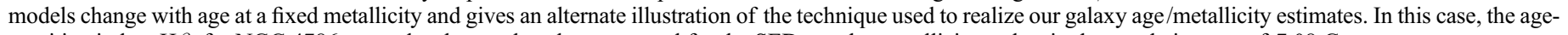
sensitive index, $\mathrm{H} \beta$, for NGC 4786 most closely matches that measured for the SED at solar metallicity and a single population age of 7.08 Gyr.

included in the Vazdekis models. Therefore, we predicted the underlying $\mathrm{H} \alpha$ absorption spectrum for a given contaminated galaxy spectrum by matching that galaxy's $\mathrm{Hn} / \mathrm{Fe}$ index with a Vazdekis model galaxy displaying a similar $\mathrm{Hn} / \mathrm{Fe}$ index. The matching Vazdekis model SED was then treated as our model absorption spectrum. Next, we smoothed this spectrum to the appropriate resolution and velocity dispersion, normalized it to zero, and subtracted it from the host-galaxy spectrum. This left the $\mathrm{H} \alpha$ emission free of any contaminating absorption. The emission-line flux was measured and converted into $\mathrm{H} \beta$ emission flux assuming a Case B Balmer decrement (Osterbrock 1989).

We used a flux-calibrated spectrum of the Orion Nebula taken with the same instrumental setup to model the emission spectrum intended to be subtracted off the contaminated galaxy. The Orion spectrum was smoothed to the appropriate velocity dispersion and scaled so that the $\mathrm{H} \beta$ flux matched the calculated value. The continuum was removed from the modified Orion spectrum, and the result was subtracted from the contaminated host-galaxy spectrum.

\section{RESULTS}

\subsection{Diagnostic Grids}

In order to measure the $\mathrm{H} \beta$, $\mathrm{Fe} \lambda 5270$, and $\mathrm{Fe} \lambda 4383$ indices, we employed the FORTRAN77 code LECTOR provided by Alexandre Vazdekis. The code measures the indices of a onedimensional input spectrum and calculates an error estimate based on photon statistics (Cardiel et al. 1998; Vazdekis \& Arimoto 1999; Cenarro et al. 2001). With the line strengths we generated our index-index diagrams, in which each vertex represents a combi- nation of line-index measurements for a single model of given age and metallicity.

A qualitative comparison between several models and a representative host galaxy are given in Figure 1, showing a comparison of the spectrum for host galaxy NGC $4786\left(Z \approx Z_{\odot}\right)$ to model SEDs with similar metallicity $\left(Z \approx Z_{\odot}\right)$ but with varying population age. Quantitative age and metallicity estimates are found through a comparison with these model SEDs by way of an interpolation of the index-index diagnostic grids. Figure 2 shows the $\mathrm{Fe} \lambda 5270-\mathrm{H} \beta$ and $\mathrm{Fe} \lambda 4383-\mathrm{H} \beta$ grids. In each case the non-emission-corrected host-galaxy indices are plotted in the upper frames, and the emission-corrected indices are plotted in the lower frames. Although for each galaxy metallicity and age interpolation we broadened the models according to their corresponding velocity dispersions, for the sake of presentation we show the whole sample plotted on a model grid broadened to a velocity dispersion of $\sigma=200 \mathrm{~km} \mathrm{~s}^{-1}$.

Computing ages and metallicities for our galaxy samples required the interpolation, and on occasion extrapolation, of an irregular grid. This was accomplished using bivariant polynomial transformations of the following form (Wolberg 1994; Cardiel 2003):

$$
\begin{aligned}
\operatorname{age}_{i} & =\sum_{j}^{N} \sum_{k}^{N-i} p_{j k}\left(\text { ind } x_{i}\right)^{j}\left(\operatorname{ind} y_{i}\right)^{k} \\
Z_{i} & =\sum_{j}^{N} \sum_{k}^{N-i} q_{j k}\left(\text { ind } x_{i}\right)^{j}\left(\operatorname{ind} y_{i}\right)^{k}
\end{aligned}
$$




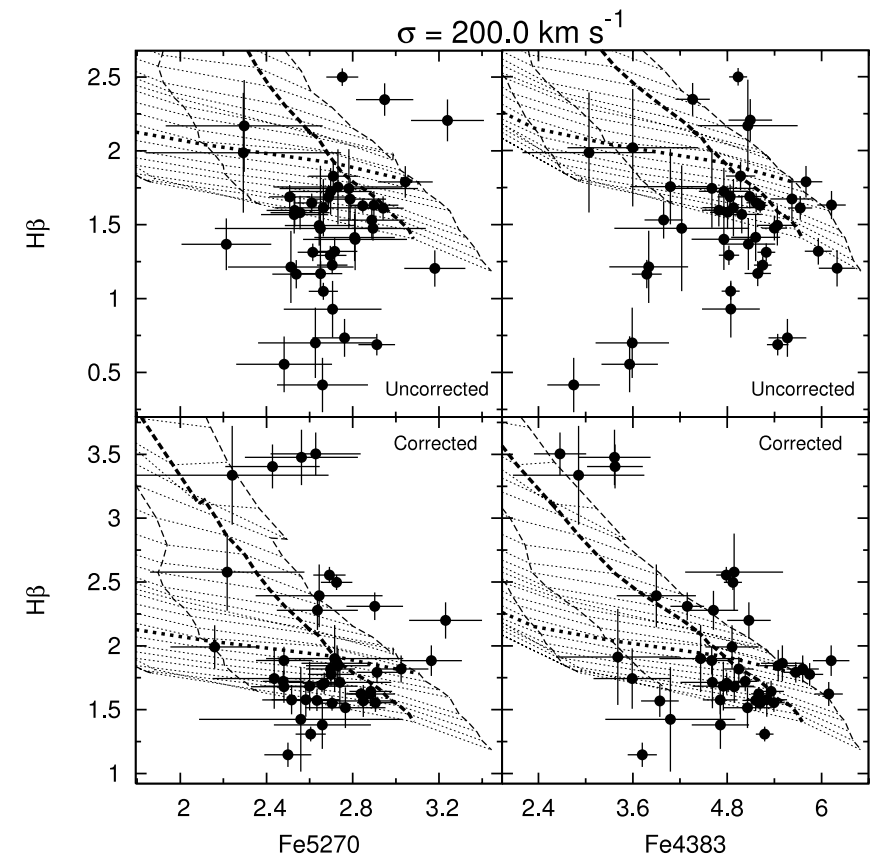

FIG. 2.-Pre- and post-emission-corrected diagnostic grids. Age-sensitive absorption-line index equivalent width $(\AA), \mathrm{H} \beta$, is plotted against the metallicitysensitive line indices $\mathrm{EW}, \mathrm{Fe} \lambda 5270$ and Fe $\lambda 4383$, before (top) and after (bottom) emission correction. The underlying grid reflect the Vazdekis model SEDs broadened to a velocity dispersion, $\sigma=200.0 \mathrm{~km} \mathrm{~s}^{-1}$. Dashed lines (near vertical) are models of common metallicity, and dotted lines (near horizontal) are those of common age. The bold dashed line identifies models of solar metallicity, and the bold dotted line marks models of age 7.08 Gyr.

where $N$ is the order of the polynomial and $p_{j k}$ and $q_{j k}$ are the coefficients that are solved for a set of nearest-neighbor points (ind $x$, ind $y$ ) indexed by $i$. We performed a second-degree approximation, thus requiring us to compute at least 12 coefficients by solving two systems of six linear equations. An IDL ${ }^{4}$ code was written that allowed us to interactively select the nearest 6-12 grid points (the number was dependent on the quality of the grid around the host galaxy's grid position), and the coefficients were computed by solving the above system of equations using the method of least squares.

The upper and lower limits for age and metallicity were determined directly from the absorption-line index limits outputted by LECTOR. The extent of the index limits can be seen from the error bars in Figure 2. The upper and lower limits were treated as unique points for which age and metallicity estimates were made. In the case of large index errors, the grid points chosen by our code were different from those used to determine the age/metallicity of the galaxy. An assumption was made that the upper and lower $\mathrm{H} \beta$ index limits corresponded to the lower and upper limits, respectively, of our galaxy age estimate. Similarly, the upper and lower limits of the Fe $\lambda 5270$ index corresponded to the upper and lower limits of the galaxy metallicity estimate. The final uncertainty was recorded as the magnitude of the difference between the age and metallicity of the limits and those of the galaxy. Furthermore, due to the irregular nature of the grid, a symmetric error in an index measurement translated into an asymmetric error in age and metallicity.

The results from our analyses are shown in Table 4. Column (1) gives the galaxy name. Columns (2)-(7) give the ages, metallicities, and corresponding errors determined via the $\mathrm{Fe} \lambda 5270-\mathrm{H} \beta$ diagram, while columns (8)-(13) give the same results ascertained via the $\mathrm{Fe} \lambda 4383-\mathrm{H} \beta$ diagram.

\subsection{Field Galaxy Sample}

Aspiring to test the uniqueness of SN Ia early-type host galaxies relative to random early-type galaxies, we compared the global properties of our SN Ia sample of elliptical host galaxies to those of a general sample of elliptical field galaxies from SDSS. The following steps were taken to generate a comparative sample of field galaxies. The SDSS Catalog Archive Server was queried for galaxy spectra within the redshift range $0 \leq z \leq 0.15$ that had a velocity dispersion measured by SDSS. The positions of these galaxies were then cross-referenced against galaxies in the NASA/ IPAC Extragalactic Database (NED), and only those galaxies characterized as E/S0 by NED were accepted. The galaxy spectra of these elliptical field galaxies were then obtained from the SDSS Data Archive Server. The spectra were emission-line corrected using a synthetic Orion spectrum created via the IRAF artificial data generation package, ARTDATA. Furthermore, rather than broadening the models to the specific velocity dispersion of each galaxy, we broadened the set of models to velocity dispersions in $30 \mathrm{~km} \mathrm{~s}^{-1}$ intervals from 100 to $340 \mathrm{~km} \mathrm{~s}^{-1}$. The set of models used in the analysis for a given SDSS galaxy spectrum was the set broadened for a velocity dispersion that most closely matched that of the galaxy. Finally, to speed up the calculation, the grid points to perform the diagnostic interpolations were automatically chosen for the SDSS sample by an algorithm designed to mimic the grid-point selection criteria used for the interactive point selection in the host-galaxy analysis.

\subsection{Data Quality and Consistency Check}

The quality of our data is assessed via three checks, the results of which are shown in Figure 3. First, we compare the respective age and metallicity estimates obtained from our two index-index diagnostics. Figure $3 a$ shows the Fe $\lambda 4383$ age estimate plotted against the age estimate of $\mathrm{Fe} \lambda 25270$. Given that both ages were determined using the same age-sensitive index, namely, $\mathrm{H} \beta$, we would expect these data to be consistent with unity. As the top left panel shows, we find moderate scatter about unity by virtue of our error estimates. Nevertheless, the data are consistent with unity, with a $\chi_{r}^{2}$ of 0.88 for the fit. Figure $3 b$ shows the metallicity comparison between our two index-index diagnostics. In this case we see more significant dispersion; however, the data are still consistent with unity given the calculated uncertainty. Consequently, as a matter of preference, the galaxy ages and metallicities used in the following analysis will be those determined from the $\mathrm{H} \beta-\mathrm{Fe} \lambda 5270$ diagnostic.

Our second quality check is a comparison of our age and metallicity estimates to those of control galaxies studied by Trager et al. (2000, hereafter T00). Our data are plotted against the data of T00 in Figures $3 c$ and $3 d$. T00 made age and metallicity measurements through a central $R_{e} / 2$ aperture, and we are reporting the "preferred" nonsolar abundance ratio (NSAR) model results (model 4). The age-sensitive line index used was $\mathrm{H} \beta$, while the two metallicity-sensitive indices were $\langle\mathrm{Fe}\rangle^{5}$ and $\mathrm{Mg} b$. Figure $3 c$ shows that the age comparison for the two studies with the dotted line indicates a model 1:1 relation. The plot shows fairly good agreement between the two studies. The $\chi_{r}^{2}$ of the $1: 1$ fit is 8.55 , although it is improved if we throw out NGC 5813 at $(8.69,24.30)$, reducing $\chi_{r}^{2}$ to 2.72 .

\footnotetext{
${ }^{4}$ Interactive Data Language: http://www.ittvis.com/idl/idl7.asp.
}

${ }^{5}\langle\mathrm{Fe}\rangle \equiv\left(\frac{1}{2}\right)(\mathrm{Fe} \lambda 5270+\mathrm{Fe} \lambda 5335)$ 
TABLE 4

Ages and Metallicities

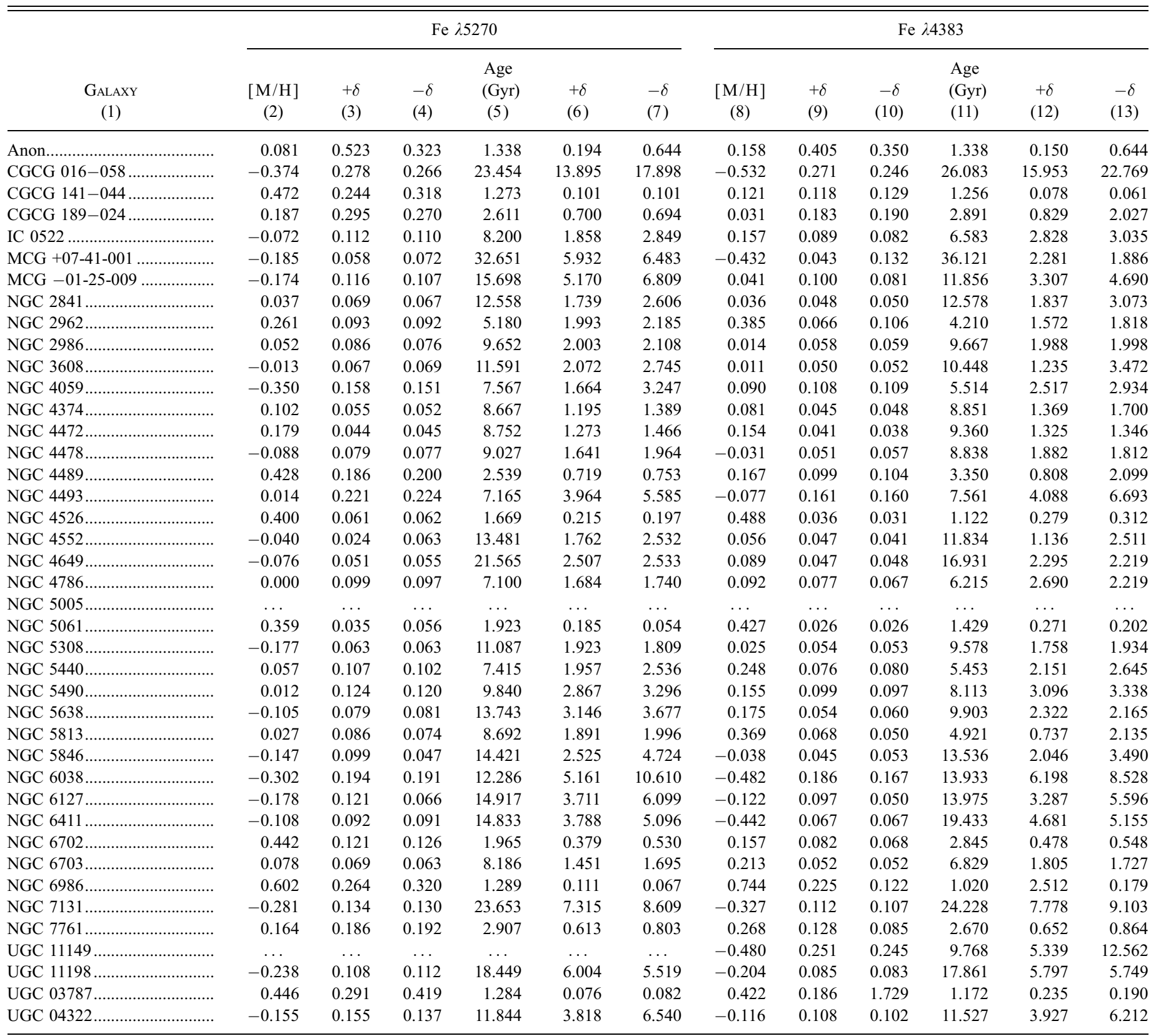

The agreement is less encouraging for the metallicity comparison in Figure $3 d$. Ignoring the few outlier points, the T00 galaxies seem to be systematically more metal-rich than those measured in our study. This is likely due to the fact that we did not account for NSAR in our analysis. Studies have shown that the abundance ratios in early-type galaxies are often nonsolar. In particular, the $\mathrm{Mg} / \mathrm{Fe}$ abundance ratio has been shown to be larger in more luminous early-type galaxies (O'Connell 1976; Peletier 1989; Worthey et al. 1992; Vazdekis et al. 1997). Given that both $\mathrm{Mg}$ and $\mathrm{Fe}$ lines strongly factored into the metallicity measurements of T00, we should not expect to see a straight 1:1 comparative ratio. Nevertheless, throwing out the points with the two greatest dispersions (NGC 5813 and NGC 4489), we seem to be measuring the relative metallicities consistently with T00, thereby enabling us to find potential trends between age/metallicity and SN Ia properties.
We also compare our results to those published in Thomas et al. (2005). In their study, they seek to set constraints on the epochs of early-type galaxy formation through an analysis of 124 early-type galaxies in both high- and low-density regions. We have 15 galaxies in common with their study and compare our $\mathrm{H} \beta$ index, age, and metallicity measurements to those from their analysis. Similar to the comparison with T00, we see general agreement between our respective results. However, the agreement is far from 1:1, with broad scatter greater than that seen in our T00 comparison. Once again, this is not entirely unexpected given our lower $\mathrm{S} / \mathrm{N}$ and the fact that we did not account for potential $\alpha$ enhancement in our galaxies. Moreover, they were sampling a significantly smaller fraction of the galactic light with an aperture radius of $R_{e} / 10$. Overall, our comparisons with both T00 and Thomas et al. (2005) show reasonable agreement and confirm the ability of our analysis 

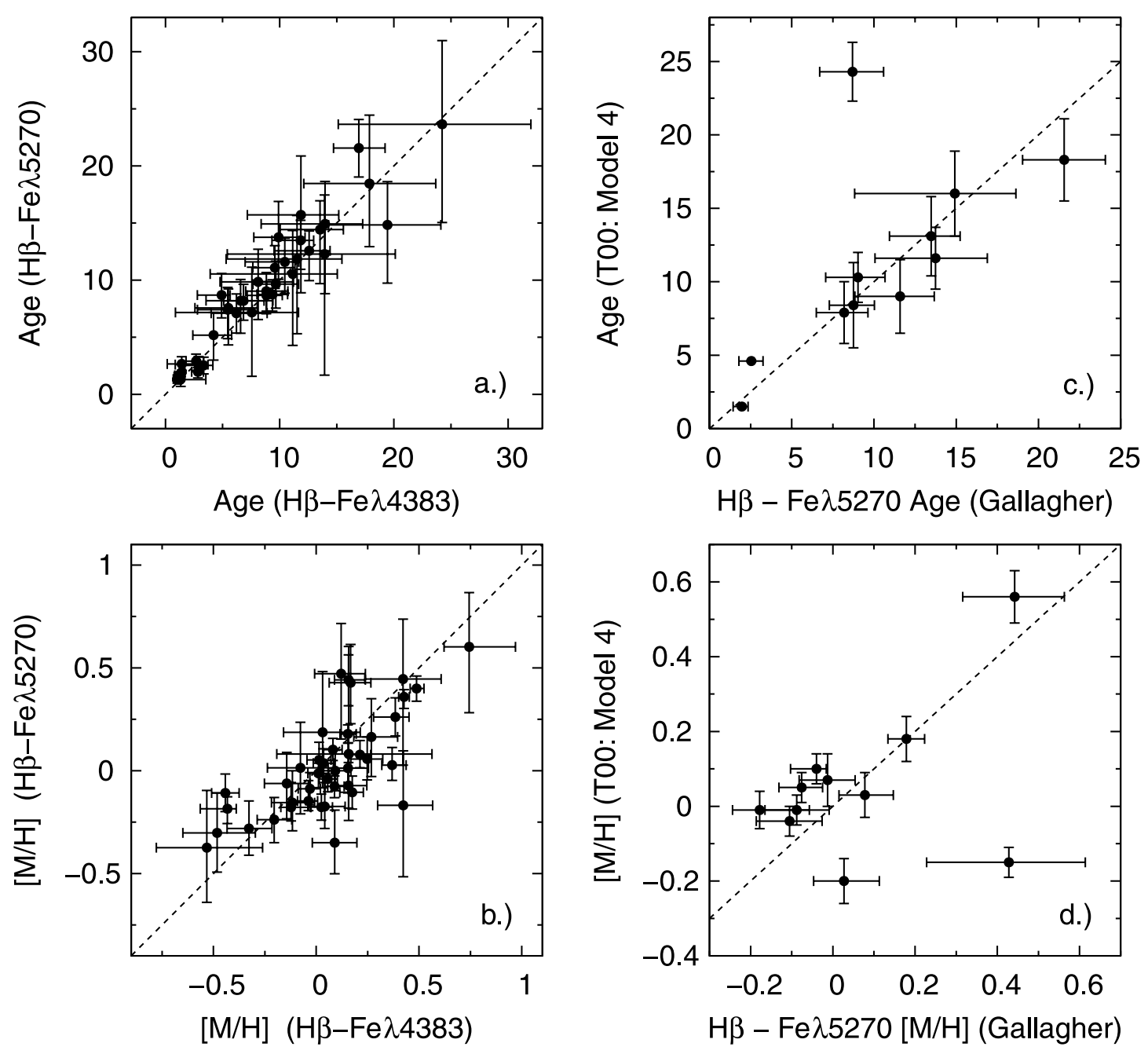

FIG. 3.-Quality and consistency check. $(a, b)$ Comparision of the age and metallicity estimates from our two diagnostic grids presented in Fig. 2, respectively. In both cases

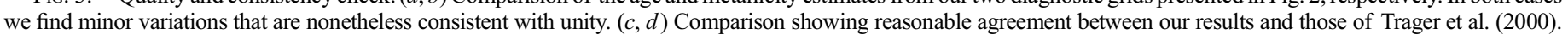

to measure acceptable relative population ages and metallicities for our SN Ia host-galaxy sample.

Finally, we compare the $B-K$ colors for the host galaxy and SDSS data to the galaxy age; see Figure 4 . The expected average $B-K$ color for a sample of elliptical galaxies is 4.0 (Jarret et al. 2003), and we can see that both our host galaxies and the SDSS galaxies meet this expectation. The figure also shows that both of the samples fall within the range predicted by the evolutionary synthesis models of Bruzual \& Charlot (2003). The middle line is the predicted trend of $B-K$ color with age for a population of solar metallicity, the top line is the prediction for a metallicity of $[\mathrm{M} / \mathrm{H}] \approx-0.68$, and the bottom line is the prediction for a metallicity of $[\mathrm{M} / \mathrm{H}] \approx 0.40$.

\subsection{Age and Metallicity}

The host-galaxy age and metallicity vs. SN Ia peak $V$-band magnitude are plotted in Figure 5. The peak magnitudes were derived from measurements of the light-curve shape parameter, $\Delta$, fitted using MLCS2k2 by the authors of Jha et al. (2007). ${ }^{6}$ In Figure $5 a$ the data show that the youngest populations tend to host the brightest SNe Ia, but with a wide spread. However, the eye has a tendency to gravitate toward large error bars, thereby placing an unwanted amount of attention to those points. Con-

\footnotetext{
${ }^{6} M_{V}($ peak $)=-19.504+0.736 \Delta+0.182 \Delta^{2}+5 \log \left(H_{0} / 65 \mathrm{~km} \mathrm{~s}^{-1} \mathrm{Mpc}^{-1}\right)$
}

sequently, we have replotted the data in Figures $5 b$ and $5 d$ with point sizes that are inversely proportional to their uncertainty. In this way, the data with the smallest uncertainty in age will be represented by the largest points. When points with the largest uncertainty are de-emphasized, the trend between age and luminosity is very clear, but it is nevertheless difficult to distinguish whether the effect is a smooth transition with age or the result of two distinct populations.

Gallagher et al. (2005a) found that only luminous SNe Ia occur in strongly star-forming hosts, while E/S0 galaxies show a wide range of SN Ia luminosities. This is confirmed in Figure 6, where we have plotted SN Ia peak magnitude vs. host-galaxy specific star formation, i.e., the SFR per stellar mass. The SFRs were calculated from the $\mathrm{H} \alpha$ emission flux using the relation of Kennicutt (1998) with the distance to each galaxy coming from the SN Ia luminosities. Filled circles represent the host galaxies from the current sample of early-type galaxies, while open circles are from the sample studied by G05. We eliminated galaxies from G05 if they were in our current sample since the $\mathrm{H} \alpha$ flux from $\mathrm{G} 05$ was uncorrected for underlying absorption. The points with arrows represent star formation upper limits in which $\mathrm{H} \alpha$ emission was buried within the noise. This trend, combined with Figure $5 b$, suggests that the age of the dominant population in E/S0 galaxies determines the resulting SN Ia peak luminosity. A correlation between SN Ia peak luminosity and SFR has also been seen in the high-redshift SNLS data (Sullivan et al. 2006), but their 


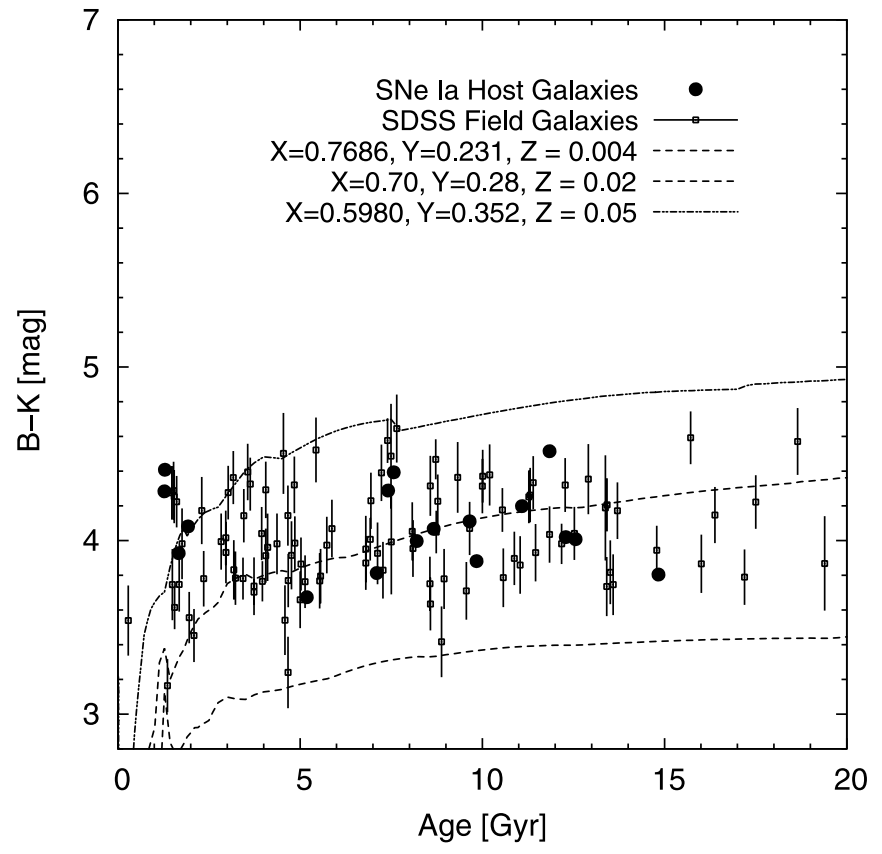

FIG. 4.-SN Ia host-galaxy $B-K$ color vs. age. We see that both of the samples fall within the range predicted by the evolutionary synthesis models of Bruzual \& Charlot (2003). The middle line is the predicted trend of $B-K$ color with age for a population of solar metallicity, the top line is the prediction for a metallicity of $[\mathrm{M} / \mathrm{H}] \approx-0.68$, and the bottom line is the prediction for a metallicity of $[\mathrm{M} / \mathrm{H}] \approx 0.40$.

type of search is biased against very low luminosity events. Our "young" E/S0 galaxies correspond to their "passive" hosts, and the extension to very old populations (with low specific SFR) confirms that population age is the major factor that determines the SN Ia peak luminosity.

The plots of host metallicity vs. SN Ia peak luminosity (Figs. $5 c$ and $5 d$ ) also show a mild correlation. This may be due to the "age/ metallicity degeneracy" shown in Figure 7. There we present measured galaxy age vs. metallicity for both the hosts and SDSS field galaxies, showing that the galaxies are not evenly distributed in the diagram but instead concentrated to the lower left half. This is simply due to the evolution of the universe that started metal-poor, meaning that there were few galaxies that are both old and metal-rich and occupy the upper right of the diagram. Thus, any correlation between age and a supernova property will result in some correlation with metallicity as well. Here the relationship between host age and SN Ia luminosity is so clear that we claim it represents a physical connection. We attribute mild effect in the metallicity relation to the lack of galaxies in the lower right quadrant of Figure $5 d$, which comes from age correlation combined with the fact that there are no old galaxies having high metal abundance.

The distributions of age and metallicity for the two samples are also shown in Figure 7, with the cumulative fraction plots shown in Figure 8. A Kolmogorov-Smirnov test reveals that there is a $63 \%$ chance that the host age distribution is drawn from the SDSS field galaxy age distribution and a $44 \%$ chance that the host metallicity distribution is drawn from the field galaxy metallicity distribution. The results suggest that, given the size of the SN Ia sample, the abundance and age of early-type galaxies that host SNe Ia are similar, on average, to normal early-type field galaxies. Assuming this, the probability of an SN Ia going off in a given early-type galaxy does not strongly depend on the age or metallicity of the galaxy.

\subsection{Hubble Residuals}

The relation correcting SN Ia peak luminosity by the lightcurve shape reduces the scatter in the low- $z$ Hubble diagram to $\sim 0.18$ mag (Jha et al. 2007). If this residual scatter is purely random, then the evolution of age and metallicity in the universe will not induce a bias in cosmological measurements with SNe Ia. However, recent studies such as Timmes et al. (2003, hereafter TBT03) and Podsiadlowski et al. (2006) have predicted that SN calibration methods should be affected by a cosmic evolution in metallicity.

In order to investigate this possible effect for $\mathrm{SNe}$ Ia hosted by early-type galaxies, we plot our measurements of age and metallicity against postcorrected SN Ia magnitude residual from the best fit to the Hubble diagram. The Hubble residual (HR) is defined as

$$
\mathrm{HR} \equiv \mu_{z}-\mu_{\mathrm{SN}}
$$

where $\mu_{z}$ is the distance modulus determined from the hostgalaxy redshift ${ }^{7}$ and $\mu_{\mathrm{SN}}$ is the distance modulus determined via the MLCS2k2-corrected SN magnitude. HR is defined in the conventional manner such that overluminous $\mathrm{SNe}$ Ia have a negative Hubble residual. ${ }^{8}$ The primary component to the uncertainty in HR comes from $\Delta \mu_{\mathrm{SN}}$. The distance errors estimated from MLCS2k2 were found to be smaller than the scatter about the Hubble diagram. The authors of Jha et al. (2007) attributed this to an intrinsic scatter in SN Ia magnitudes and accordingly imposed a constant additive correction to these distances. Given that we are probing for systematic changes in this intrinsic scatter with age and metallicity, we have not applied this correction to $\Delta \mu_{\mathrm{SN}}$.

The top left panel of Figure 9 shows the Hubble residual vs. host-galaxy metallicity along with a least-squares fit to the data (solid line). We overplotted the trend predicted by TBT03, showing at least qualitatively that the observed trend is consistent with that expected by TBT03. The top right panel shows the results of the significance analysis for our linear fit. For each point $\left([\mathrm{M} / \mathrm{H}]_{i}\right.$, $\mathrm{HR}_{i}$ ), the Hubble residual was replaced by a randomly selected HR from a Gaussian distribution centered on the actual HR with a standard deviation equal to $\Delta \mathrm{HR}$. The same was done for each metallicity using the corresponding $[\mathrm{M} / \mathrm{H}]_{i}$ and $\Delta[\mathrm{M} / \mathrm{H}]_{i}$. We performed 100,000 iterations, measured the best-fit slope $(\beta)$ to the new data each time, and generated the $\beta$ distribution for the test. The results are seen in the top right panel and show that a nocorrelation result $(\beta=0)$ is ruled out at the $98 \%$ confidence level. A similar correlation between dust/extinction and Hubble residual is not seen for our sample, thereby removing the possibility of this correlation being a secondary dust effect.

Assuming that the least-squares fit to the top left panel of Figure 9 adequately represents the trend of HR with metallicity, we would predict a 0.26 mag increase in intrinsic SN Ia magnitude per unit decline in progenitor (galaxy) metallicity. We have looked at several studies, two theoretical and one observational, that seek to characterize cosmic chemical evolution. The theoretical studies by Cen \& Ostriker (1999) and Cen et al. (2003) predict an approximate 0.05 and 0.25 dex drop in metallicity per unit redshift, respectively. The observational study conducted by Kewley \& Kobulnicky (2007) finds a preliminary result of $0.15 \mathrm{dex} / z$.

\footnotetext{
7 Imposed lower redshift cutoff at $2500 \mathrm{~km} \mathrm{~s}^{-1}$ to decrease uncertainty due to peculiar velocities.

${ }^{8}$ Note that the opposite definition was adopted by Gallagher et al. (2005a).
} 

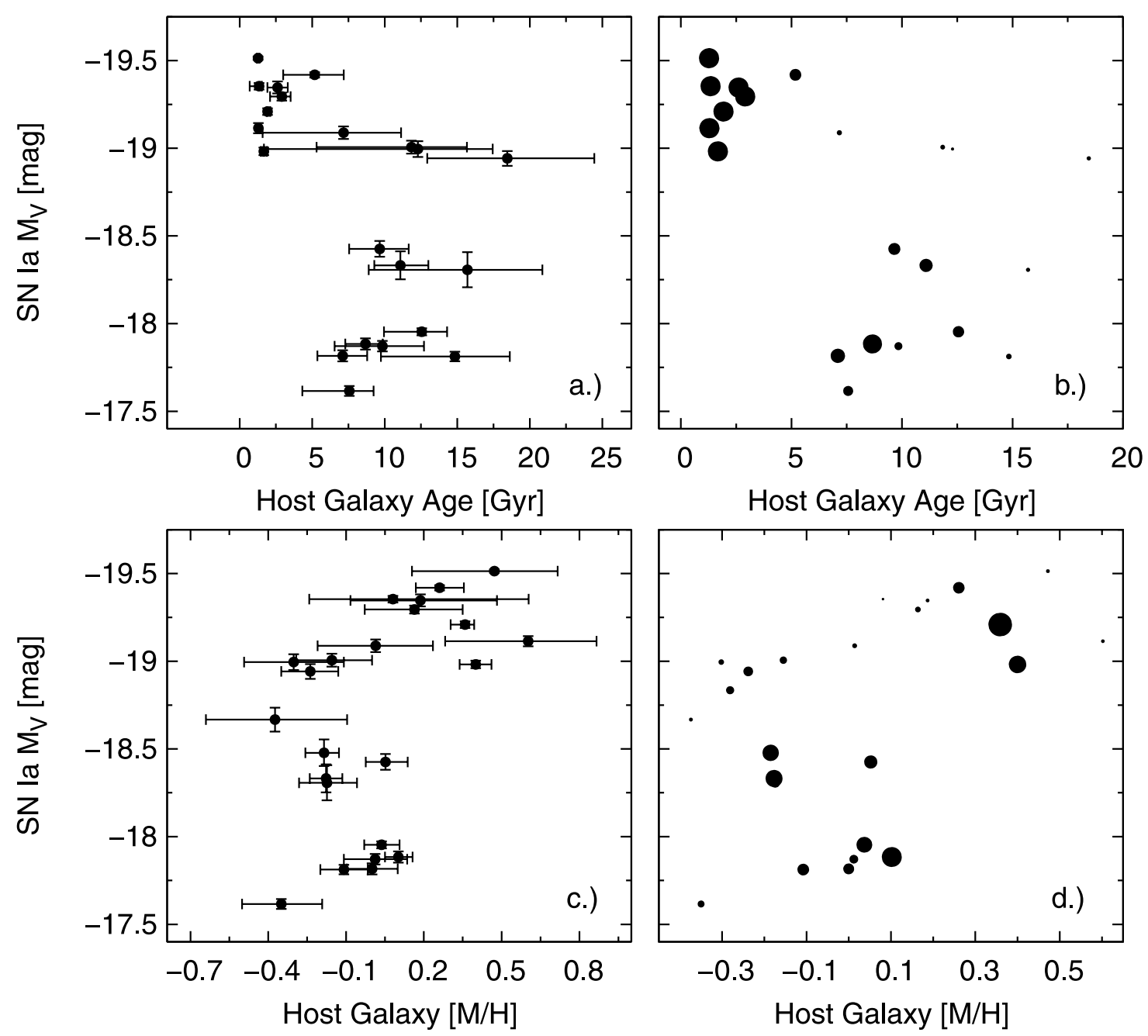

FIG. 5.-SN Ia peak magnitudes $\left(H_{0}=72 \mathrm{~km}^{-1} \mathrm{~s}^{-1} \mathrm{Mpc}^{-1}\right)$ vs. (a) luminosity-weighted host-galaxy age and $(c)$ metallicity. $(b, d)$ Same data but with the point size reflecting the average error along the abscissa. Point size is inversely proportional to the average error along the abscissa, with the exception of our young hosts in $(b)$ where an upper limit is imposed on the point size. The data in $(b)$ show a clear trend between SN Ia peak magnitude and host-galaxy age. The behavior could be evidence of either a smooth transition of SN magnitude with age or of two distinct populations of SNe Ia. $(d)$ Less convincing correlation, likely arising from the degeneracy between age and metallicity.

Taking the average of these three studies $(0.15 \mathrm{dex} / z)$ for the true decline in metallicity with redshift would translate into a 0.039 mag decrease in intrinsic SN Ia magnitude per unit redshift. Assuming an ideal SN Ia data set out to $z<1.8$, a $\chi^{2}$ minimization of three parameters $\left(\Omega_{M}, w \text {, and } H_{0}\right)^{9}$ applied to a set of model SNe Ia subject to this $4 \%$ systematic error would induce an approximate $9 \%$ systematic error on the measurement of the equation-of-state parameter $w=P /\left(\rho c^{2}\right)$.

Jha et al. (2007) showed that the scatter of SN magnitudes about the Hubble diagram is lower in $\mathrm{E} / \mathrm{S} 0$ galaxies $(\sim 0.13 \mathrm{mag})$ than for the sample as a whole ( $\sim 0.18 \mathrm{mag})$. Our sample of E/S0 hosts show a scatter of approximately 0.14 mag. By applying a correction to SN magnitudes consistent with our least-squares fit, we reduce the scatter about the Hubble diagram to approximately $0.11 \mathrm{mag}$, illustrating the potential for such a correction to significantly improve SN Ia distance measurements.

The bottom left panel of Figure 9 shows the age of the parent galaxy vs. the Hubble residual of the SN Ia. The plot does not reveal a significant trend of HR with host-galaxy age. Indeed, the probability of a zero-correlation result is only ruled out at the $56 \%$ confidence level, suggesting that SN Ia magnitudes corrected for

\footnotetext{
${ }^{9}$ Flat geometry assumed.
}

light-curve shape are likewise being corrected for any age bias. It should be noted that two galaxies (CGCG 016-058 and MCG +07-41-001) were removed from the age plot because their positions on their respective diagnostic grids required a difficult extrapolation that rendered their age estimates extremely uncertain.

\subsection{SN Ia Rate vs. Population Age}

Since the late 1970s, observations have shown that SNe Ia are more prevalent in star-forming late-type galaxies than in earlytype galaxies (Oemler \& Tinsley 1979). This fact has been confirmed again and again with new studies showing that the SN Ia rate per unit mass is significantly higher in blue/late-type galaxies than in red/early-type galaxies (van den Bergh 1990; Della Valle \& Livio 1994; Mannucci et al. 2005; J. Leaman et al. 2008, in preparation). The current explanation for these observations is that there are prompt and delayed (tardy) SN Ia explosions. The prompt component is dependent on the rate of recent star formation, and the delayed component is dependent on the total number of low-mass stars. The combination of these two components is believed to orm the overall observed SN Ia rate (Scannapieco \& Bildsten 2005; Mannucci et al. 2005; Sullivan et al. 2006; J. Leaman et al. 2008, in preparation). 


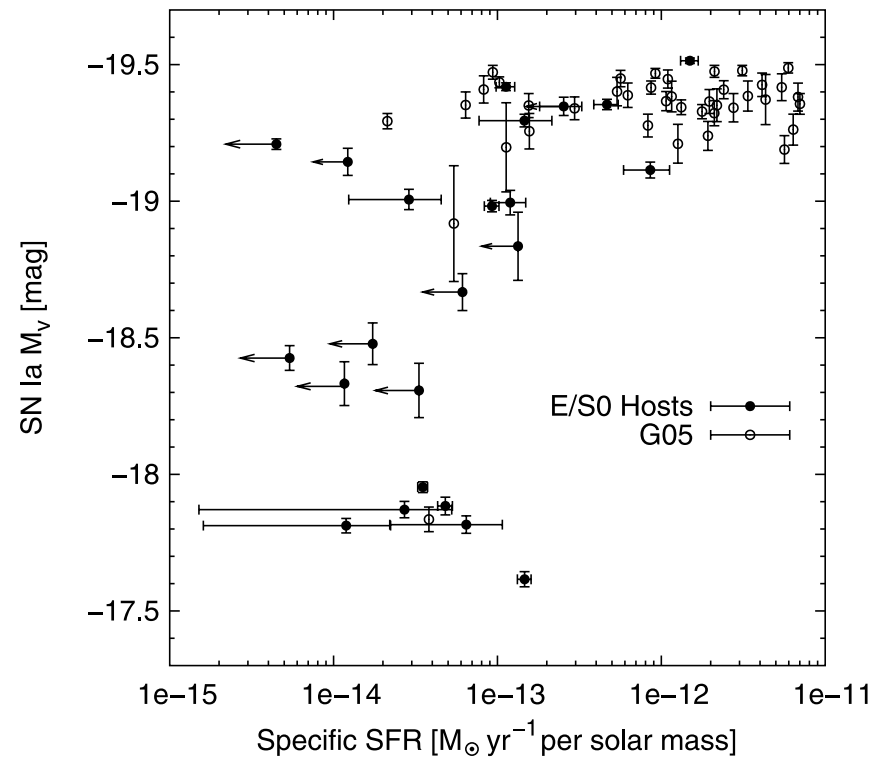

FIG. 6.-Dependence of SN Ia peak magnitude on host-galaxy specific $\mathrm{SFR}_{\mathrm{H} \alpha}$. Filled circles are the current sample of early-type hosts, while open circles represent the high-SFR hosts of G05. The points marked by arrows indicate upper limits. On average, our specific SFR distribution is lower than that seen in the sample of high-z passive hosts of Sullivan et al. (2006). However, aside from the expected decrease of SFR over cosmological time, it should be noted that our data only sample half of the galactic light given our extraction radius $\left(R_{e}\right)$, and the SFRs for the passive galaxies of Sullivan et al. (2006) were randomly assigned a rate of $\sim 0.005 \mathrm{M}_{\odot} \mathrm{yr}^{-1}$.

Sullivan et al. (2006) investigated the parameters shaping the overall SN Ia rate by observing the relative presence of SNe Ia in high- $z$ host galaxies of differing mass and SFR. Our direct age measurement means that we can do a similar analysis at low redshifts by estimating the relative SN Ia rate as a function of host-galaxy age. Figures 10 and 11 summarize the details of our analysis on the relative SN Ia rate in elliptical host galaxies of various ages.

The panel on the left of Figure 10 contains two unique histograms. The dotted histogram represents the number of host galaxies, or equivalently the number of SNe Ia, per age bin (identical to the distribution in Fig. 7 with decreased bin size). The solid

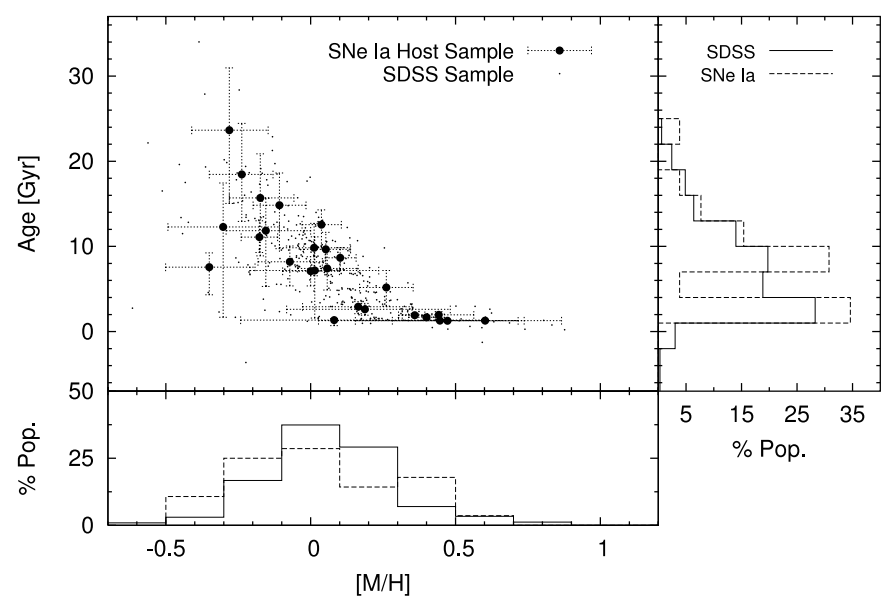

FIG. 7.- Illustration of the age-metallicity degeneracy "problem" in the study of stellar populations. The plot shows the luminosity-weighted age vs. metallicity results for our sample of SN Ia host galaxies and SDSS elliptical field galaxies. The plot reveals the trend stretching from young, metal-rich to old, metal-poor stellar populations. Histograms are also presented illustrating the age and metallicity distributions for the two respective samples.
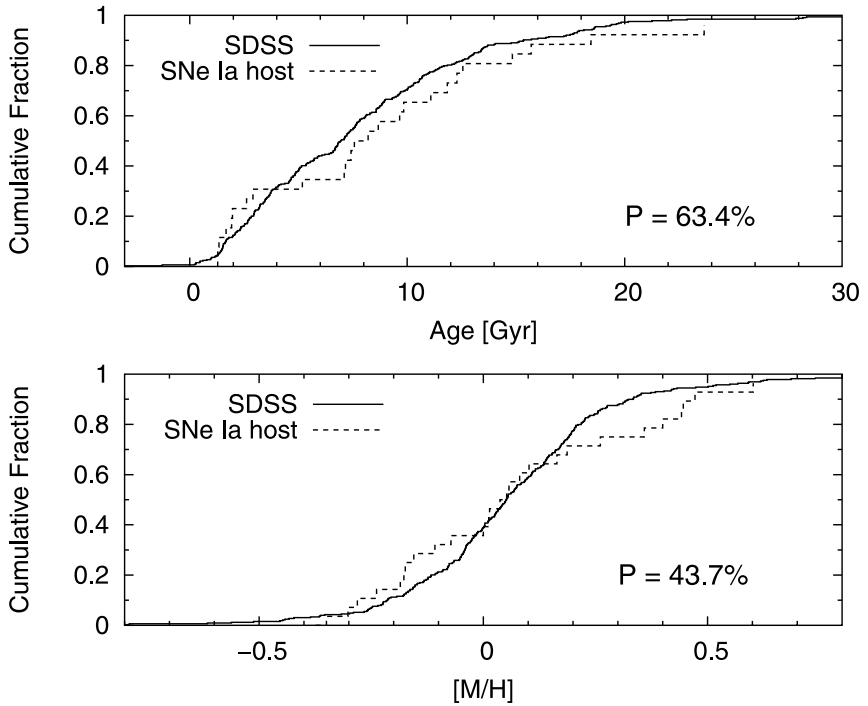

FIG. 8.-Cumulative fraction plots of the SN Ia host galaxy and SDSS galaxy age and metallicity distributions. Our sample of SN Ia host galaxies is clearly similar in both age and metallicity to the SDSS field galaxy sample. We find a $63 \%$ and $44 \%$ probability that the SDSS age and metallicity distributions, respectively, represent acceptable parent populations from which the host metallicities were drawn.

histogram is the expected number of stars per age bin. Each of the distributions has been normalized such that the bin encompassing $8 \mathrm{Gyr}$ contains exactly one element. The number of stars is derived from the $K$-band SDSS galaxy distribution, the $K$ band being a relatively accurate tracer of stellar mass. $K$-band apparent magnitudes for the SDSS sample were compiled from NED, and the distances to the galaxies in the sample were calculated from the SDSS redshift measurements, where care was taken to exclude those galaxies with $v_{r}<2500 \mathrm{~km} \mathrm{~s}^{-1}$. The $K$-band absolute magnitudes, $M_{g}$, were derived from the distances and apparent magnitudes. Finally, the number of stars per galaxy was approximated by the expression

$$
N_{\text {stars }}=100^{\left[\left(M_{K, \odot}-M_{g}\right) / 5.0\right]} .
$$

The variable $M_{K, \odot}$ is the corresponding absolute magnitude of the Sun in the $K$ band. ${ }^{10}$ The number of stars per age bin was then calculated by summing the stars in each galaxy in each age bin.

The relative SN Ia rate per unit stellar mass is defined as the ratio of the number of SNe per age bin to the expected number of stars per age bin. The panel on the right of Figure 10 shows the expected SN Ia rate per unit stellar mass as a function of age. The plot predicts a rate that is high for young galaxies, falls for intermediate-age galaxies, and increases and moderates for older populations. This result is consistent with the "dual-component model," given that the SN rate at low age could arise from a short delay time population of $\mathrm{SNe}$ Ia predicted to occur in galaxies with high SFR, and the rise in SN rate at late age could likely result from the predicted rise in SN rate with stellar mass (Sullivan et al. 2006). However, we take the less general route and suggest the possibility that the rate at low population age is proportional to the rate of WD production following an initial burst of star formation. This function can be seen in Figure 11. Similarly, the latetime SN rate can be traced by the cumulative number of WDs

\footnotetext{
${ }^{10} M_{K, \odot}=3.28$.
} 

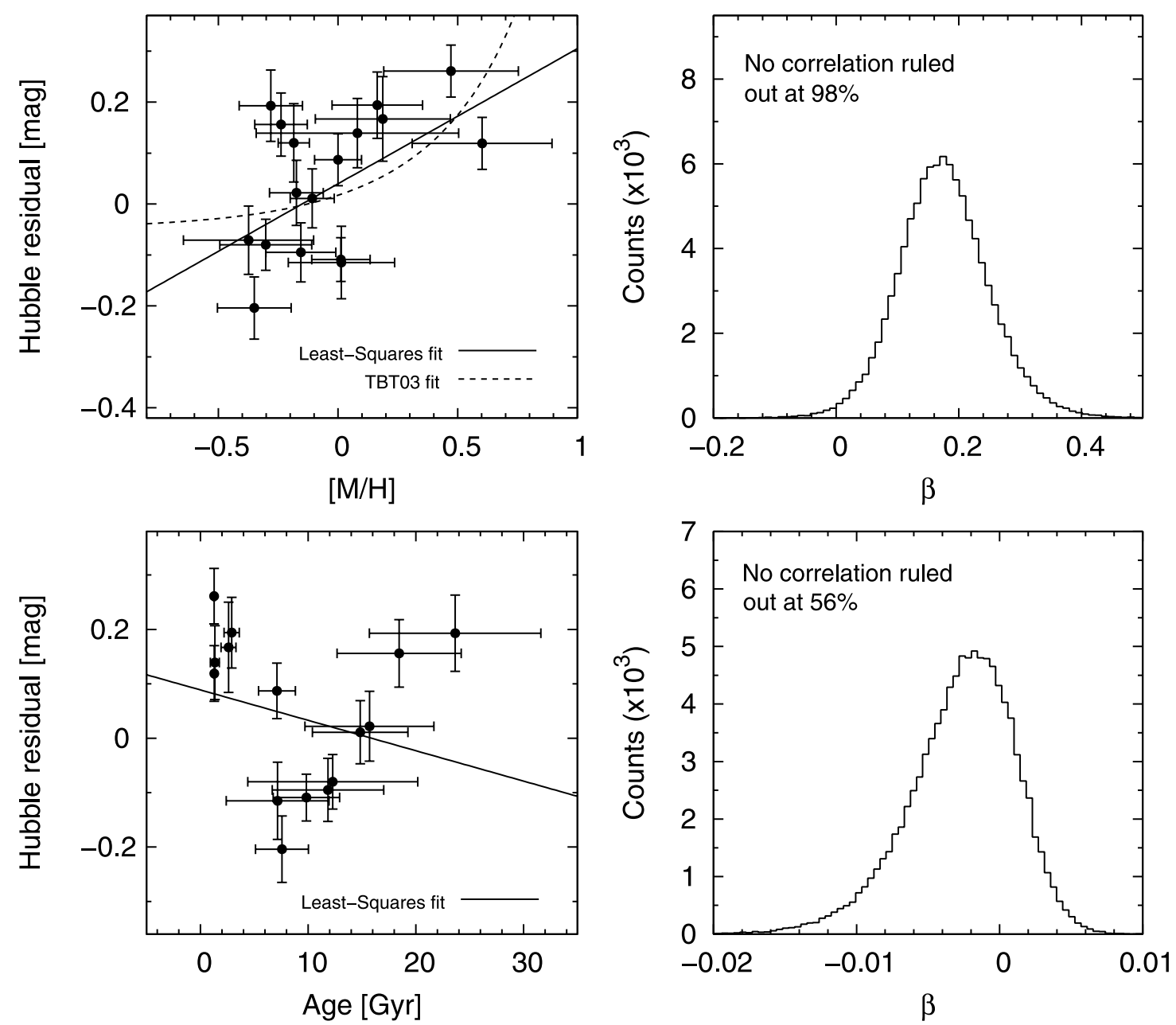

FIG. 9.-SN Ia Hubble residual vs. luminosity-weighted metallicity (top left) and age (bottom left). A general trend is found suggesting that more metal-rich populations produce intrinsically fainter SNe Ia. A least-squares fit to the data places a slope for the trend at 0.26 . The dotted line represents the predicted trend based on the analytical models of Timmes et al. (2003). The results of our statistical tests are presented in the adjacent plots. We rule out a no-correlation result for the HR vs. metallicity data at the $98 \%$ confidence level. A least-squares fit to the HR vs. age plot yields a nominal slope of -0.03 with negligible significance.

present in the galaxy (Fig. 11). This value is determined for an age $t=t_{0}$ by integrating the WD production rate from time $t=0$ to $t=t_{0}$. These components of the SN Ia rate are related to those of Sullivan et al. (2006), and yet they specifically target the population of stars believed to be the progenitors of SN Ia explosions.
An important caveat to this analysis is that we are only sampling the dominant stellar population within a given host galaxy. It has been shown that a significant portion (15\%-30\%) of elliptical and lenticular (S0) galaxies show evidence of recent star formation (Yi et al. 2005; Kaviraj et al. 2007), which has the
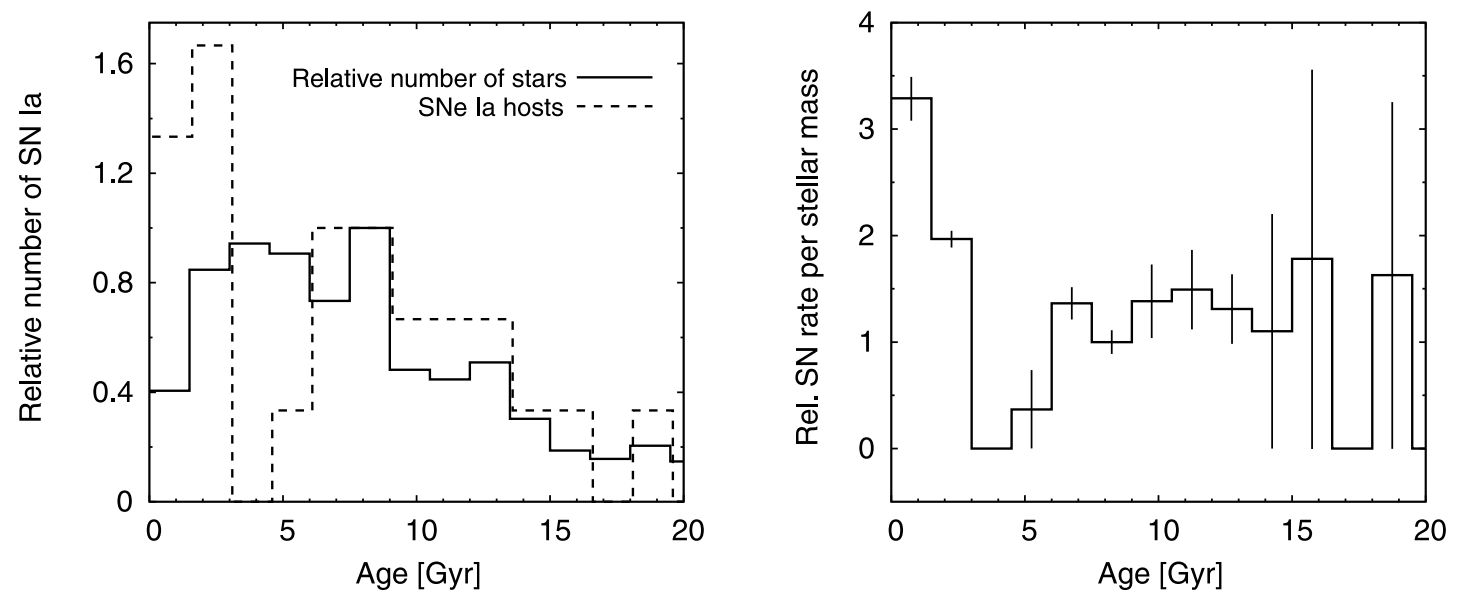

FIG. 10.- Relative SN Ia rate per stellar mass as a function of age in early-type galaxies. Stellar masses were approximated from each galaxy's $K$-band luminosity.

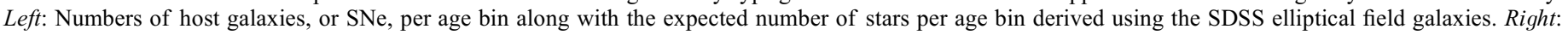
Expected relative SN Ia rate per age bin, showing a consistency with the dual-component model. 


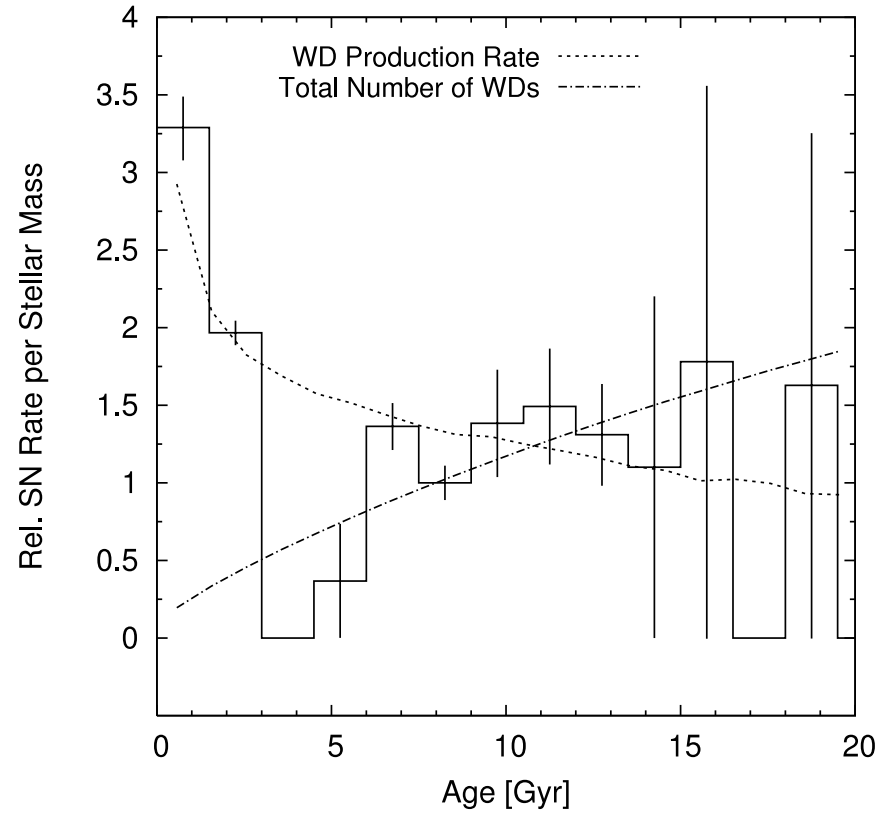

FIG. 11.-Relative SN Ia rate per stellar mass is replotted, showing the bimodal nature of the distribution. The dotted line represents the rate of white dwarf production, while the dash-dotted line is the accumulated number of white dwarfs as a function of time since the burst.

potential to compromise our conclusion that SNe Ia exploding in old stellar populations possess longer delay times.

\section{SUMMARY AND CONCLUSIONS}

We have analyzed a sample of early-type galaxies that have hosted SNe Ia. Comparing the data with stellar population synthesis models, we have measured the global age and metallicity of the SN Ia elliptical host galaxies and a general sample of elliptical galaxies from the SDSS. Our results indicate that there is likely a significant correlation between age or metallicity and $\mathrm{SN}$ Ia absolute magnitude. Given the mounting evidence of an
SN Ia rate dependence on specific SFR, we find it most likely that the SN Ia peak magnitudes are correlated with age, and the observed trend with metallicity is merely an artifact brought about by the evolutionary entanglement of age and metallicity.

We find the global distributions of age and metallicity to be similar to those of our SDSS elliptical galaxy sample, suggesting that the presence, or absence, of an SN Ia in an elliptical galaxy is not dependent on either age or metallicity. Moreover, we detect a trend between early-type host-galaxy metallicity and the residuals from the Hubble diagram at the $98 \%$ confidence level. This trend is consistent with the predictions of TBT03 and with a trend observed for late-type galaxies by Gallagher et al. (2005); it suggests that metal-rich galaxies produce underluminous $\mathrm{SNe} I \mathrm{I}$, even after correcting for their light-curve shapes. Furthermore, we conclude that the failure to apply a metallicity correction to SN Ia magnitudes could potentially introduce a $9 \%$ error into current and future measurements of $w$.

Finally, we find the predicted SN Ia rate as a function of age for our sample of early-type galaxies and determine it to be moderately consistent with the "dual-component model" of Sullivan et al. (2006). Also, we describe our preferred model governing the SN Ia rate in which the "prompt" component is proportional to the WD production rate and the "delayed" component scales with the cumulative WD population.

Partial funding for this work came through NASA LTSA grant NAG5-9364. Supernova research at Harvard University is supported by NSF Grant AST 06-06772. A.V. F.'s supernova group at the University of California, Berkeley is supported by NSF grant AST 06-07485, as well as by the TABASGO Foundation. KAIT was made possible by generous donations from Sun Microsystems, Inc., the Hewlett-Packard Company, AutoScope Corporation, Lick Observatory, the NSF, the University of California, and the Sylvia and Jim Katzman Foundation. We thank Sumner Starrfield and the Arizona State University for granting us access to their computer network that proved invaluable for the completion of this work.
Astier, P., et al. 2006, A\&A, 447, 31

Barris, B. J., et al. 2004, ApJ, 602, 571

Bennett, C. L., et al. 2003, ApJS, 148, 1

Blakeslee, J. P., Vazdekis, A., \& Ajhar, E. A. 2001, MNRAS, 320, 193

Bressan, A., Chiosi, C., \& Tantalo, R. 1996, A\&A, 311, 425

Bruzual, G., \& Charlot, S. 2003, MNRAS, 344, 1000

Buzzoni, A. 1995, ApJS, 98, 69

Caldwell, N., Rose, J. A., \& Concannon, K. D. 2003, AJ, 125, 2891

Cardelli, J. A., Clayton, G. C., \& Mathis, J. S. 1989, ApJ, 345, 245

Cardiel, N. 2003, A\&A, 409, 511

Cardiel, N., Gorgas, J., Cenarro, J., \& Gonzalez, J. J. 1998, A\&AS, 127, 597

Cen, R., \& Ostriker, J. P. 1999, ApJ, 519, L109

Cen, R., Ostriker, J. P., Prochaska, J. X., \& Wolfe, A. M. 2003, ApJ, 598, 741

Cenarro, A. J., et al. 2001, MNRAS, 326, 959 2007, MNRAS, 374, 664

Conley, A., et al. 2008, ApJ, 681, 482

Della Valle, M., \& Livio, M. 1994, ApJ, 423, L31

Eisenstein, D. J., Seo, H, Sirko, E., \& Spergel, D. N. 2007, ApJ, 664, 675

Fabricant, D., Cheimets, P., Caldwell, N., \& Geary, J. 1998, PASP, 110, 79

Faber, S. M. 1972, A\&A, 20, 361

Filippenko, A. V. 1982, PASP, 94, 715

2005a, in ASP Conf. Ser. 332, The Fate of the Most Massive Stars, ed.

R. Humphreys \& K. Stanek (San Francisco: ASP), 33

2005b, in White Dwarfs: Cosmological and Galactic Probes, ed. E. M.

Sion, S. Vennes, \& H. L. Shipman (Dordrecht: Springer), 97

Filippenko, A. V., Li, W., Treffers, R. R., \& Modjaz, M. 2001, in IAU Colloq.

183, Small-Telescope Astronomy on Global Scales, ed. W.-P. Chen, C. Lemme,

\& B. Paczyński (ASP Conf. Ser. 246; San Francisco: ASP), 121
REFERENCES

Fioc, M., \& Rocca-Volmerange, B. 1997, A\&A, 326, 950

Freedman, W. L., et al. 2001, ApJ, 553, 47

Frieman, J. A., Turner, M., \& Huterer, D. 2008, ARA\&A, in press (arXiv:0803.0982)

Frieman, J. A., et al. 2007, ApJ, submitted (arXiv:0708.2749)

Gallagher, J. S., Garnavich, P. M., Berlind, P., Challis, P., Jha, S., \& Kirshner, R. P. 2005a, ApJ, 634, 210 (G05)

Gallagher, J. S., Garnavich, P. M., Modjaz, M., Kirshner, R. P., \& Challis, P. 2005b, IAU Circ., 8514, 3

Ganeshalingam, M., et al. 2008, in preparation

Garnavich, P. M., et al. 1998a, ApJ, 493, L53

. 1998b, ApJ, 509, 74

Goldhaber, G., et al. 2001, ApJ, 558, 359

González Delgado, R. M., Cerviño, M., Martins, L. P., Leitherer, C., \& Hauschildt, P. H. 2005, MNRAS, 357, 945

Gunn, J. E., Stryker, L. L., \& Tinsley, B. M. 1981, ApJ, 249, 48

Guy, J., et al. 2007, A\&A, 466, 11

Hamuy, M., Phillips, M. M., Suntzeff, N. B., Schommer, R. A., Maza, J., \& Avilles, R. 1996, AJ, 112, 2391

Hamuy, M., et al. 2000, AJ, 120, 1479

Hicken, M., et al. 2007, ApJ, 669, L17

Höflich, P., Wheeler, J. C., \& Thielemann, F. K. 1998, ApJ, 495, 617

Howell, A. D., et al. 2006, Nature, 443, 308

Iben, I., \& Tutukov, A. V. 1984, ApJS, 54, 335

Jarret, T. H., Chester, T., Cutri, R., Schneider, S. E., \& Huchra, J. P. 2003, AJ, 125,525

Jha, S. 2002, Ph.D. thesis, Harvard Univ.

Jha, S., Riess, A. G., \& Kirshner, R. P. 2007, ApJ, 659, 122

Jha, S., et al. 1999, ApJS, 125, 73 
Jones, T. M. 1997, Ph.D. thesis, Univ. North Carolina, Chapel Hill

Kaviraj, S., et al. 2007, ApJS, 173, 619

Kennicutt, R. C., Jr. 1998, ARA\&A, 36, 189

Kewley, L., \& Kobulnicky, H. A. 2007, in Island Universes (New York: Springer), 435

Kirshner, R. P. 2002, The Extravagant Universe: Exploding Stars, Dark Energy, and the Accelerating Cosmos (Princeton: Princeton Univ. Press)

Knop, R. A., et al. 2003, ApJ, 598, 102

Krisciunas, K., et al. 2007, AJ, 133, 58

Mannucci, F., et al. 2005, A\&A, 433, 807

Maraston, C. 1998, MNRAS, 300, 872

Miknaitis, G., et al. 2007, ApJ, 666, 674

Nomoto, K. 1982, ApJ, 253, 798

Norgaard-Neilsen, H. U., et al. 1989, Nature, 339, 523

O’Connell, R. W. 1976, ApJ, 206, 370

Oemler, A., \& Tinsley, B. M. 1979, AJ, 84, 985

Osterbrock, D. E. 1989, Astrophysics of Gaseous Nebulae and Active Galactic Nuclei (Mill Valley: University Science)

Peacock, J. A., et al. 2001, Nature, 410, 169

Peletier, R. F. 1989, Ph.D. thesis, Univ. Groningen

Perlmutter, S., et al. 1997, ApJ, 483, 565

$$
\text { 1998, Nature, 391, } 51
$$$$
\text { 1999, ApJ, 517, } 565
$$

Phillips, M. M. 1993, ApJ, 413, L105

Phillips, M. M., Lira, P., Suntzeff, N. B., Schommer, R. A., Hamuy, M., \& Maza, J. 1999, AJ, 118, 1766

Podsiadlowski, P., Mazzali, P. A., Lesaffre, P., Wolf, C., \& Forster, F. 2006, preprint (astro-ph/0608324)

Renzini, A. 2006, ARA\&A, 44, 141

Riess, A. G., Press, W. H., \& Kirshner, R. P. 1996, ApJ, 473, 88

Riess, A. G., et al. 1998, AJ, 116, 1009 2004, ApJ, 607, 665 2005, ApJ, 627, 579 2007, ApJ, 659, 98

Salpeter, E. E. 1955, ApJ, 121, 161

Sanchez-Blazquez, P., et al. 2006, MNRAS, 371, 703
Sandage, A., Tammann, G. A., Saha, A., Reindl, B., Macchetto, F. D., \& Panagia, N. 2006, ApJ, 653, 843

Scannapieco, E., \& Bildsten, L. 2005, ApJ, 629, L85

Schmidt, B. P., et al. 1998, ApJ, 507, 46

Soubiran, C., Katz, D., \& Cayrel, R. 1998, A\&AS, 133, 221

Sullivan, M., et al. 2006, ApJ, 648, 868

Taubenberger, S., et al. 2008, MNRAS, 385, 75

Thomas, D., Maraston, C., Bender, R., \& Mendes de Oliveira, C. 2005, ApJ, 621,673

Timmes, F. X., Brown, E. F., \& Truran, J. W. 2003, ApJ, 590, L83 (TBT03)

Tinsley, B. M. 1980, Fundam. Cosmic Phys., 5, 287

Tinsley, B. M., \& Gunn, J. E. 1976, ApJ, 203, 52

Tonry, J. L., et al. 2003, ApJ, 594, 1

Trager, S. C., Faber, S. M., Worthey, G., \& Gonzales, J. J. 2000, AJ, 119, 1645 ( T00)

Umeda, H., Nomoto, K., Kobayashi, C., Hachisu, I., \& Kato, M. 1999, ApJ, 522, L43

van den Bergh, S. 1990, PASP, 102, 1318

Vazdekis, A. 1999, ApJ, 513, 224

Vazdekis, A., \& Arimoto, N. 1999, ApJ, 525, 144

Vazdekis, A., Cenarro, A. J., Gargas, J., Cardiel, N., \& Peletier, R. F. 2003, MNRAS, 340, 1317

Vazdekis, A., Peletier, R. F., Beckman, J. E., \& Casuso, E. 1997, ApJS, 111, 203

Vazdekis, A., et al. 1996, ApJS, 106, 307

Vázquez, G. A., \& Leitherer, C. 2005, ApJ, 621, 695

Webbink, R. F. 1984, ApJ, 277, 355

Whelan, J., \& Iben, I. J. 1973, ApJ, 186, 1007

Wolberg, G. 1994, Digital Image Warping (Washington: IEEE Computer Society Press)

Wood-Vasey, W. M., et al. 2007, ApJ, 666, 694

2008, ApJ, submitted (arXiv:0711.2068)

Worthey, G. 1994, ApJS, 95, 107

Worthey, G., Faber, S. M., \& Gonzalez, J. J. 1992, ApJ, 398, 69

Worthey, G., \& Ottaviani, D. L. 1997, ApJS, 111, 377

Yi, S. K., et al. 2005, ApJ, 619, L111 